\title{
Novel delay-dependent robust stability criteria for neutral-type time-varying uncertain Lurie nonlinear control system with mixed time delays
}

\author{
Kaibo Shi ${ }^{\mathrm{a}}$, Youhua Wei ${ }^{\mathrm{b}, *}$, Shouming Zhong ${ }^{\mathrm{c}}$, Jun Wang ${ }^{\mathrm{d}}$ \\ a School of Information Science and Engineering, Chengdu University, Chengdu, 610106, China. \\ ${ }^{b}$ College of Geophysics, Geomathematics Key Laboratory of Sichuan Province, Chengdu University of Technology, Chengdu, 610059, \\ China. \\ ${ }^{c}$ School of Mathematics Sciences, University of Electronic Science and Technology of China, Chengdu Sichuan, 611731, China. \\ ${ }^{d}$ College of Electrical and Information Engineering, Southwest University for Nationalities, Chengdu, 610041, China.
}

Communicated by $\mathrm{X}$. Liu

\begin{abstract}
This study examines the problem of robust stability analysis of neutral-type time-varying uncertain Lurie nonlinear control system with mixed time delays. Firstly, by discretizing the time-delay interval into non-uniformly multiple subintervals and decomposing the corresponding integral intervals to estimate the bounds of integral terms more exactly, less conservative stability criteria are derived. Secondly, based on the above delay-partitioning method, a newly augmented Lyapunov-Krasovkii functional is constructed. Thirdly, by taking full advantage of Wirtinger's integral inequality, which can provide tighter upper bound than Jensen's inequality, novel delay-dependent robust stability conditions are obtained in terms of linear matrix inequalities. Finally, several numerical examples are presented to illustrate the effectiveness and advantages of the theoretical results. (C)2017 All rights reserved.
\end{abstract}

Keywords: Lurie nonlinear control system, mixed time-varying delays, Wirtinger's integral inequality, Lyapunov-Krasovkii functional, linear matrix inequality.

2010 MSC: 93C42, 93D20.

\section{Introduction}

During the past few decades, the study of time-delay systems (TDSs) has attracted increasing attention due to the fact that time delay is an unavoidable factor in a variety of physical and engineering problems, and may lead to instability, poor performance or even oscillation $[3,7,29,31]$. Therefore, the issue of stability analysis for TDSs has become a popular subject of research for their extensive applications in practical systems, such as $\mathrm{H}_{\infty}$ out tracking control system [36], markovian jump system [32], $\mathrm{H}_{\infty}$ filtering [4], reliable passive control for singular systems [28], dissipativity analysis [38], neural networks [22, 23], and other scientific areas.

\footnotetext{
*Corresponding author

Email address: weiyouhua@cdut.cn (Youhua Wei)
}

doi:10.22436/jnsa.010.04.71 
To date, TDSs are often classified into two categories, namely, retarded type $[2,26,30,35]$ and neutral type $[1,8,10,12-14,16-18,25,27,37,39]$. The retarded type system only depends on the state delay, while the neutral type system not only relies on its state, but also its derivatives of state. TDSs can be frequently found in a large amount of dynamical systems, for example, population ecology, distributed networks containing lossless transmission lines, the control of constrained manipulators with delay measurements and so on. Due to their extensive applications in practice, the stability analysis for neutral-type systems have received considerable interest in the recent. Meanwhile, numerous important and interesting research results on the delayed stability analysis have been also proposed. In [30], the authors have investigated the problem of state robust $\mathrm{H}_{\infty}$ tracking control for uncertain stochastic systems with interval time-varying delay by employing reciprocally convex approach. In [26], by introducing a parameter $\lambda$ in the Lyapunov-Krasovkii functional (LKF), new delay-derivation-dependent stability conditions have been derived in term of (linear matrix inequalities) LIMs. The problem of exponential stability for uncertain neutral switched systems with nonlinear perturbations has been studied in [37]. In order to improve further the feasible region of stability criteria, many effective methods have been developed, such as slack matrices or free-weighting matrices [2,12], reciprocally convex [5], delay-partitioning [1, 14], state matrix decomposition [17], model transformation [27], a multiple integral inequality [7, 23].

On the other hand, it is well-known that many nonlinear systems could be modeled in the form of Lurie control system (LCS), such as Chua's circuit, Hyper chaotic attractors and Lorenz system, which consist of a feedback connection of a linear system and a nonlinear element satisfying the sector condition. However, in practice, stability of TDSs may be frequently destroyed by its nonlinear perturbations. Therefore, a good deal of effort has been paid to the stability analysis of DLCS in the past decades $[5,6,9,11,15,19-21,24,33,34]$. Based on LKF approach, less conservative synchronization criteria have been obtained in [11]. The authors in $[5,20,33]$ have considered the problem of delay-dependent stability of a class for uncertain NTLCS with time-varying delays and sector-bounded nonlinearity via some effective mathematical techniques. The design of a PD controller for robust $\mathrm{H}_{\infty}$ stabilization of uncertain LCS with sector and slope restricted nonlinearities has been studied by using convex properties of the nonlinearities in [34]. By introducing an appropriate LKF and utilizing second order reciprocal convex combination technique, improved delay-dependent stability criteria have been derived in terms of LMIs in [15, 24]. Paper in [19] has proposed a new method to study LCS with mixed time-varying delays. That is dividing the time-varying delay interval $[0, h(t)]$ into multiple subintervals with the same size. The method may take fully the information about the delay interval $[0, h]$ into account, which can reduce the conservativeness of results. To the best of our knowledge, there are still some information of nonlinear functions which have not been well utilized in $[5,6,9,11,15,19-21,24,33,34]$, which may lead to the conservatism of proposed results to a certain extent. Therefore, it is a challenging and valuable issue to develop many more effective methods with less conservative results.

Motivated by the issues discussed above, the issue of delay-dependent robust stability for time-varying uncertain neutral-type Lurie nonlinear control system (NTLNCS) with mixed time-varying delays is studied by using some new approaches in this paper. The main contributions of this paper are listed in the following. In the first place, we propose a new delay-partitioning method (DPM) which is more general than the one in [19]. The method is: dividing the time-varying delay interval $[0, h]$ into non-uniformly multiple subintervals $\sum_{i=1}^{N}\left[\rho_{i-1} h, \rho_{i} h\right]$. In the second place, a novelly augmented LKF is constructed by choosing various weighting matrices corresponding to different subsegments. In the third place, improved results are derived by making the best of the Wirtinger's integral inequality (WII), which may provide tighter upper bound than Jensen's integral inequality. Finally, five numerical examples are presented to show the improvements over the existing results and the effectiveness of the proposed methods.

Notation: Notations used in this paper are fairly standard: $\mathbb{R}^{\mathfrak{n}}$ denotes the $n$-dimensional Euclidean space; $\mathbb{R}^{n \times m}$ is the set of all $n \times m$ dimensional matrices; I denotes the identity matrix of appropriate dimensions; $\mathbf{A}^{\top}$ stands for the matrix transposition of the matrix $\mathbf{A}$. By $\mathbf{X} \succ 0$ (respectively $\mathbf{X} \succeq 0$ ), for $\mathbf{X} \in \mathbb{R}^{\mathfrak{n} \times n}$, we mean that the matrix $\mathbf{X}$ is real symmetric positive definite (respectively, positive semidefinite); $\operatorname{diag}\left\{r_{1}, \cdots, r_{n}\right\}$ diagonal matrix with diagonal elements $r_{i}, i=1, \cdots, n$, the symbol $*$ represents 
the elements below the main diagonal of a symmetric matrix; $\langle\mathbf{M}\rangle_{\mathrm{s}}$ is defined as $\langle\mathbf{M}\rangle_{\mathrm{s}}=\frac{1}{2}\left(\mathbf{M}+\mathbf{M}^{\top}\right) ; \rho(\cdot)$ denotes the spectral radius of given matrix.

\section{Preliminaries}

Consider the following time-varying uncertain NTLNCS with mixed time-varying delays and sectorbounded nonlinearity:

$$
\left\{\begin{array}{l}
\dot{\mathbf{x}}(t)-\mathbf{C} \dot{\mathbf{x}}(t-\tau(t))=(\mathbf{A}+\triangle \mathbf{A}(t)) \mathbf{x}(t)+\left(\mathbf{A}_{d}+\triangle \mathbf{A}_{d}(t)\right) \mathbf{x}(t-h)+(\mathbf{D}+\triangle \mathbf{D}(t)) \mathbf{f}(\sigma(t)) \\
\sigma(t)=\mathbf{H}^{\top} \mathbf{x}(t)=\left[\mathbf{h}_{1}, \cdots, \mathbf{h}_{m}\right]^{\top} \mathbf{x}(t), \quad t \geqslant 0 \\
\mathbf{x}(t)=\phi(t), \quad t \in[-\max \{h, \tau\}, 0]
\end{array}\right.
$$

where $\mathbf{x}(t) \in \mathbb{R}^{n}$ is the state vector, $\sigma(t) \in \mathbb{R}^{m}$ is the output vector, $\mathbf{A} \in \mathbb{R}^{\mathfrak{n} \times n}, \mathbf{A}_{d} \in \mathbb{R}^{n \times n}, D \in$ $\mathbb{R}^{n \times m}, C \in \mathbb{R}^{n \times n}, H \in \mathbb{R}^{n \times m}$ are known real matrices, $\mathbf{f}(\sigma(t)) \in R^{m}$ is the nonlinear function in the feedback path. Its form is formulated as

$$
\left\{\begin{array}{l}
\mathbf{f}(\sigma(t))=\left[f_{1}\left(\sigma_{1}(t)\right), \cdots, f_{m}\left(\sigma_{m}(t)\right)\right]^{\top} \\
\sigma(t)=\left[\sigma_{1}(t), \cdots, \sigma_{m}(t)\right]^{\top}=\mathbf{H}^{\top} \mathbf{x}(t)=\left[\mathbf{h}_{1}, \cdots, \mathbf{h}_{m}\right]^{\top} x(t),
\end{array}\right.
$$

wherein, each term $f_{s}\left(\sigma_{s}(t)\right), s=1, \cdots, m$, satisfies one of the following sector conditions:

$$
f_{s}\left(\sigma_{s}(t)\right) \in K_{\left[0, k_{s}\right]}=\left\{f_{s}\left(\sigma_{s}(t)\right) \mid f_{s}(0)=0,0<\sigma_{s}(t) f_{s}\left(\sigma_{s}(t)\right) \leqslant k_{s} \sigma_{s}^{2}(t), \sigma_{s}(t) \neq 0\right\},
$$

or

$$
f_{s}\left(\sigma_{s}(t)\right) \in K_{[0, \infty]}=\left\{f_{s}\left(\sigma_{i}(t)\right) \mid f_{s}(0)=0, \sigma_{s}(t) f_{s}\left(\sigma_{s}(t)\right)>0, \sigma_{s}(t) \neq 0\right\}
$$

$h$ and $\tau(t)$ are constant discrete delay and time-varying neutral delay, respectively. $\tau(t)$ satisfies the following conditions:

$$
0 \leqslant \tau(t) \leqslant \tau, \quad \dot{\tau}(t) \leqslant \tau_{c}<1,
$$

where $\tau$ and $\tau_{c}$ are constants, $\phi(t)$ is initial condition.

In addition, $\triangle \mathbf{A}(t), \triangle \mathbf{A}_{d}(t)$ and $\triangle \mathbf{D}(\mathrm{t})$ are time-varying uncertain matrices of appropriate dimensions, which are assumed to be of the following form:

$$
\left[\triangle \mathbf{A}(\mathrm{t}), \triangle \mathbf{A}_{\mathrm{d}}(\mathrm{t}), \triangle \mathbf{D}(\mathrm{t})\right]=\mathbf{N F}(\mathrm{t})\left[\mathrm{E}_{1}, \mathrm{E}_{2}, \mathrm{E}_{3}\right],
$$

where $\mathbf{N}, \mathbf{E}_{1}, \mathbf{E}_{2}$ and $\mathbf{E}_{3}$ are known real constant matrices of appropriate dimensions, and $\mathbf{F}(\mathbf{t})$ is a timevarying uncertain matrix satisfying

$$
\mathbf{F}(\mathbf{t})^{\mathrm{T}} \mathbf{F}(\mathbf{t}) \leqslant \mathbf{I}, \quad \forall \mathrm{t} \geqslant 0
$$

By using Eq. (2.3), the time-varying uncertain system (2.1) can be rewritten as follows:

$$
\begin{aligned}
\dot{\mathbf{x}}(\mathrm{t}) & =(\mathbf{A}+\triangle \mathbf{A}(\mathrm{t})) \mathbf{x}(\mathrm{t})+\left(\mathbf{A}_{\mathrm{d}}+\triangle \mathbf{A}_{\mathrm{d}}(\mathrm{t})\right) \mathbf{x}(\mathrm{t}-\mathrm{h})+\mathbf{C} \dot{\mathbf{x}}(\mathrm{t}-\tau(\mathrm{t}))+(\mathbf{D}+\triangle \mathbf{D}(\mathrm{t})) \mathbf{f}(\sigma(\mathrm{t})) \\
& =\mathbf{A x}(\mathrm{t})+\mathbf{A}_{\mathrm{d}} \mathbf{x}(\mathrm{t}-\mathrm{h})+\mathbf{C} \dot{\mathbf{x}}(\mathrm{t}-\tau(\mathrm{t}))+\mathbf{D} \mathbf{f}(\sigma(\mathrm{t}))+\mathbf{N P}(\mathrm{t})
\end{aligned}
$$

where $\mathbf{P}(t)=\mathbf{F}(t) \xi \vartheta(t), \xi=[\mathbf{E}_{1}, 0,0,0, \mathbf{E}_{3}, \overbrace{0, \cdots, 0}^{N-1}, \mathbf{E}_{2}, \overbrace{0, \cdots, 0}^{N}, \vartheta^{\top}(t)=\left[\mathbf{x}(t)^{\top}, \dot{\mathbf{x}}^{\top}(t), \mathbf{x}(t-\tau(t))^{\top}, \dot{\mathbf{x}}^{\top}(t-\right.$ $\tau(t)), \mathbf{f}^{\top}(\sigma(t)), \mathbf{x}^{\top}\left(t-\rho_{1} h\right), \cdots, x^{\top}\left(t-\rho_{j} h\right), \cdots, x^{\top}\left(t-\rho_{N-1} h\right), x^{\top}(t-h), \varpi_{1}^{\top}(t), \cdots, \varpi_{j}^{\top}(t), \cdots, \varpi_{N+1}^{\top}(t)$, $\mathbf{P}(t)], \varpi_{j}^{\top}(t)=\frac{1}{\left(\rho_{j}-\rho_{j-1}\right) h} \times \int_{t-\rho_{j} h}^{t-\rho_{j-1} h} \mathbf{x}^{\top}(s) d s, j=1, \cdots, N$. 
In order to improve the feasible region of stability criteria, by applying the idea of non-uniformly dividing delay interval, the interval $[0, h]$ is divided into $N$ variable subintervals. That is, dividing $[0, h]$ into $N$ segments $\sum_{j=1}^{N}\left[\rho_{j-1} h, \rho_{j-1} h\right] . \rho_{i}$ is the predetermined parameter satisfying the following conditions:

$$
0=\rho_{0} h<\cdots<\rho_{i} h<\cdots<\rho_{N} h=h, \quad \rho_{0}=0, \quad \rho_{N}=1, \quad \rho_{i} \in(0,1) \quad(i=1, \cdots, N-1) .
$$

The following lemma is introduced, which will be used in the proof of the main results.

Lemma 2.1 ([35]). For a given symmetric positive definite matrix $\mathbf{R}>0$, and for differentiable signal $\mathbf{x}(\mathbf{t})$ in $[\alpha, \beta] \rightarrow \mathbb{R}^{n}$, the following inequality holds:

$$
-(\beta-\alpha) \int_{\alpha}^{\beta} \dot{\mathbf{x}}^{\top}(\mathrm{s}) \mathrm{R} \dot{\mathbf{x}}(\mathrm{s}) \mathrm{d} s \leqslant-\left[\begin{array}{c}
\mathbf{x}(\alpha) \\
\mathbf{x}(\beta) \\
\frac{1}{\beta-\alpha} \int_{\alpha}^{\beta} \mathbf{x}(\mathrm{s}) \mathrm{ds}
\end{array}\right]^{\top}\left[\begin{array}{ccc}
4 \mathbf{R} & 2 \mathbf{R} & -6 \mathbf{R} \\
* & 4 \mathbf{R} & -6 \mathbf{R} \\
* & * & 12 \mathbf{R}
\end{array}\right]\left[\begin{array}{c}
\mathbf{x}(\alpha) \\
\mathbf{x}(\beta) \\
\frac{1}{\beta-\alpha} \int_{\alpha}^{\beta} \mathbf{x}(s) \mathrm{ds}
\end{array}\right],
$$

where $\alpha$ and $\beta$ are two real constants.

\section{Stability analysis in the finite sector $\mathrm{K}_{\left[0, \mathrm{k}_{s}\right]}$}

In this section, we will propose a novel delay-dependent stability criterion of NTLNCS (2.1) via using a general and complete DPM. Firstly, we consider the case in which the nonlinearity $f_{s}\left(\sigma_{s}(t)\right)$ satisfy the sector condition:

$$
f(\sigma(t)) \in K_{\left[0, k_{s}\right]}(s=1, \cdots, m) .
$$

For the sake of simplicity of matrix representation, $\mathbf{e}_{i}^{\top}=[\overbrace{0, \cdots, 0}^{i}, \mathbf{I}, \overbrace{0, \cdots, 0}^{2 \mathrm{~N}+6-i}](i=1, \cdots, 2 \mathrm{~N}+6), \hat{\mathbf{e}}_{i}^{\top}=$ $\overbrace{0, \cdots, 0}^{i}, \mathbf{I}, \overbrace{0, \cdots, 0}^{2 \mathrm{~N}+4-i}(i=1, \cdots, 2 \mathrm{~N}+4)$ and $\tilde{\mathbf{e}}_{i}^{T}=[\overbrace{0, \cdots, 0}^{i}, \mathbf{I}, \overbrace{0, \cdots, 0}^{2 \mathrm{~N}+5-i}(i=1, \cdots, 2 \mathrm{~N}+5)$ are defined as block entry matrices.

Theorem 3.1. For given positive scalars $\tau, \tau_{c}, h$ and $\varepsilon>0$, the time-varying uncertain NTLNCS (2.1) with nonlinearity located in the finite sector $\mathrm{K}_{\left[0, \mathrm{k}_{s}\right]}$ is globally asymptotically stable if $\rho(\mathbf{C})<1$ and there exist positive definite matrices $\mathbf{P}>0, \mathbf{W}>0, \mathbf{P}_{\mathfrak{i}}>0, \mathbf{R}_{\mathbf{j}}>0, \mathbf{W}_{\mathbf{j}}>0(\boldsymbol{i}=1,2 ; \mathbf{j}=1, \cdots, \mathbf{N})$, any positive definite diagonal matrices $\mathbf{G}=\operatorname{diag}\left\{g_{1}, \cdots, g_{m}\right\} \geqslant 0, \mathbf{L}=\operatorname{diag}\left\{l_{1}, \cdots, l_{m}\right\} \geqslant 0, \mathbf{B}=\operatorname{diag}\left\{b_{1}, \cdots, b_{m}\right\} \geqslant 0$, and arbitrary matrices $\mathbf{T}_{1}, \mathbf{T}_{2}$ and $\mathbf{T}_{3}$ with appropriate dimensions, such that the following LIM holds:

$$
\Sigma<0,
$$

where

$$
\begin{aligned}
& \Sigma=\mathbf{e}_{1}\left(\mathbf{P}_{1}+\mathbf{R}_{1}-\frac{1}{\tau} \mathbf{W}-\frac{4}{\rho_{1} h} \mathbf{W}_{1}+\left\langle\mathbf{T}_{2} \mathbf{A}\right\rangle_{\mathrm{s}}\right) \mathbf{e}_{1}^{\top} \\
& +\mathbf{e}_{2}\left(\mathbf{P}_{2}+\tau \mathbf{W}+\rho_{1} h \mathbf{W}_{1}+\sum_{i=1}^{N-1}\left(\rho_{i+1}-\rho_{i}\right) h \mathbf{W}_{i+1}-\left\langle\mathbf{T}_{1}\right\rangle_{s}\right) \mathbf{e}_{2}^{\top} \\
& -\mathbf{e}_{3}\left(\left(1-\tau_{c}\right) \mathbf{P}_{1}+\frac{1}{\tau} \mathbf{W}\right) \mathbf{e}_{3}^{\top}-\left(1-\tau_{c}\right) \mathbf{e}_{4} \mathbf{P}_{2} \mathbf{e}_{4}^{\top} \\
& +\mathbf{e}_{5}\left\langle\mathbf{T}_{3} \mathbf{D}-\mathbf{2} \mathbf{B}\right\rangle_{\mathrm{s}} \mathbf{e}_{5}^{\mathrm{T}}-\frac{4}{\rho_{1} \mathrm{~h}} \mathbf{e}_{6} \mathbf{W}_{1} \mathbf{e}_{6}^{\mathrm{T}}-\mathbf{e}_{5+\mathrm{N}} \mathbf{R}_{\mathrm{N}} \mathbf{e}_{5+\mathrm{N}}^{\mathrm{T}} \\
& -\frac{12}{\rho_{1} h} \mathbf{e}_{6+N} W_{1} \mathbf{e}_{6+N}^{\top}+\left\langle\mathbf{e}_{1}\left(\mathbf{P}+\mathbf{H K L H} \mathbf{H}^{\top}+\mathbf{A}^{\top} \mathbf{T}_{1}^{\top}-\mathbf{T}_{2}\right) \mathbf{e}_{2}^{\top}+\mathbf{e}_{1} \frac{1}{\tau} \mathbf{W e}_{3}^{\top}+\mathbf{e}_{1} T_{2} \mathbf{C e}_{4}^{\top}+\mathbf{e}_{1}\left(\mathbf{T}_{2} \mathbf{D}+\mathbf{A}^{\top} \mathbf{T}_{3}^{\top}\right.\right. \\
& +\mathbf{H K B}) \mathbf{e}_{5}^{\top}-\frac{2}{\rho_{1} \mathrm{~h}} \mathbf{e}_{1} \mathbf{W}_{1} \mathbf{e}_{6}^{\top}+\mathbf{e}_{1} \mathbf{T}_{2} \mathbf{A}_{\mathrm{d}} \mathbf{e}_{5+\mathrm{N}}^{\mathrm{T}}+\frac{6}{\rho_{1} \mathrm{~h}} \mathbf{e}_{1} \mathbf{W}_{1} \mathbf{e}_{6+\mathrm{N}}^{\top}+\mathbf{e}_{2} \mathbf{T}_{1} \mathbf{C e}_{4}^{\top}+\mathbf{e}_{2}\left(\mathbf{H}(\mathbf{G}-\mathbf{L})+\mathbf{T}_{1} \mathbf{D}-\mathbf{T}_{3}^{\top}\right) \mathbf{e}_{5}^{\top}
\end{aligned}
$$




$$
\begin{aligned}
& +\mathbf{e}_{2} \mathbf{T}_{1} \mathbf{A}_{\mathrm{d}} \mathbf{e}_{5+\mathrm{N}}^{\top}+\mathbf{e}_{4} \mathbf{C}^{\top} \mathbf{T}_{3}^{\top} \mathbf{e}_{5}^{\top}+\mathbf{e}_{5} \mathbf{T}_{3} \mathbf{A}_{\mathrm{d}} \mathbf{e}_{5+\mathrm{N}}^{\top}+\frac{6}{\rho_{1} \mathrm{~h}} \mathbf{e}_{6} \mathbf{W}_{1} \mathbf{e}_{6+\mathrm{N}}^{\top}+\left(\mathbf{e}_{2} \mathbf{T}_{1}+\mathbf{e}_{1} \mathbf{T}_{2}+\mathbf{e}_{5} \mathbf{T}_{3}\right) \mathbf{N} \mathbf{e}_{2 \mathrm{~N}+6}^{\top} \\
& +\sum_{i=1}^{N-1} \frac{6}{\left(\rho_{i+1}-\rho_{i}\right) h} \mathbf{e}_{5+i} \mathbf{W}_{\mathfrak{i}+1} \mathbf{e}_{6+\mathrm{N}+\mathrm{i}}^{\mathrm{T}} \\
& \left.+\sum_{i=1}^{N-1} \frac{6}{\left(\rho_{i+1}-\rho_{i}\right) h} \mathbf{e}_{6+i} \mathbf{W}_{i+1} \mathbf{e}_{6+N+i}^{T}-\sum_{i=1}^{N-1} \frac{2}{\left(\rho_{i+1}-\rho_{i}\right) h} \mathbf{e}_{5+i} \mathbf{W}_{i+1} \mathbf{e}_{6+i}^{T}\right\rangle_{s} \\
& +\sum_{i=1}^{N-1} \mathbf{e}_{i+5}\left[\mathbf{R}_{i+1}-\mathbf{R}_{i}\right] \mathbf{e}_{i+5}^{\top}-\sum_{i=1}^{N-1} \frac{4}{\left(\rho_{i+1}-\rho_{i}\right) h} \mathbf{e}_{5+i} \mathbf{W}_{i+1} \mathbf{e}_{5+i}^{\top}-\sum_{i=1}^{N-1} \frac{4}{\left(\rho_{i+1}-\rho_{i}\right) h} \mathbf{e}_{6+i} \mathbf{W}_{i+1} \mathbf{e}_{6+i}^{\top} \\
& -\sum_{i=1}^{\mathrm{N}-1} \frac{12}{\left(\rho_{i+1}-\rho_{i}\right) \mathrm{h}} \mathbf{e}_{6+\mathrm{N}+\mathrm{i}} \mathbf{W}_{\mathrm{i}+1} \mathbf{e}_{6+\mathrm{N}+\mathrm{i}}^{\mathrm{T}}+\epsilon \xi^{\top} \xi-\epsilon \mathbf{e}_{2 \mathrm{~N}+6} \mathbf{I}_{2 \mathrm{~N}+6}^{\top} .
\end{aligned}
$$

Proof. Consider an appropriate of LKF for the time-varying uncertain NTLNCS (2.1) as follows:

$$
\mathbf{V}\left(\mathbf{x}_{\mathrm{t}}\right)=\mathbf{V}_{1}\left(\mathbf{x}_{\mathrm{t}}\right)+\mathbf{V}_{2}\left(\mathbf{x}_{\mathrm{t}}\right)+\mathbf{V}_{3}\left(\mathbf{x}_{\mathrm{t}}\right)+\mathbf{V}_{4}\left(\mathbf{x}_{\mathrm{t}}\right)+\mathbf{V}_{5}\left(\mathbf{x}_{\mathbf{t}}\right),
$$

where

$$
\begin{aligned}
& \mathbf{V}_{1}\left(\mathbf{x}_{\mathrm{t}}\right)=\mathbf{x}^{\top}(\mathrm{t}) \mathbf{P} \mathbf{x}(\mathrm{t})+\int_{\mathrm{t}-\tau(\mathrm{t})}^{\mathrm{t}} \mathbf{x}^{\top}(\mathrm{s}) \mathbf{P}_{1} \mathbf{x}(\mathrm{s}) \mathrm{d} s+\int_{\mathrm{t}-\tau(\mathrm{t})}^{\mathrm{t}} \dot{\mathbf{x}}^{\top}(\mathrm{s}) \mathbf{P}_{2} \dot{\mathbf{x}}(\mathrm{s}) \mathrm{d} s, \\
& \mathbf{V}_{2}\left(\mathbf{x}_{\mathrm{t}}\right)=\int_{\mathrm{t}-\rho_{1} \mathrm{~h}}^{\mathrm{t}} \mathbf{x}^{\top}(\mathrm{s}) \mathbf{R}_{1} \mathbf{x}(\mathrm{s}) \mathrm{d} s+\sum_{i=1}^{\mathrm{N}-2} \int_{\mathrm{t}-\rho_{i+1} \mathrm{~h}}^{\mathrm{t}-\rho_{\mathrm{i}} \mathrm{h}} \mathbf{x}^{\top}(\mathrm{s}) \mathbf{R}_{\mathrm{i}+1} \mathbf{x}(\mathrm{s}) \mathrm{d} s+\int_{\mathrm{t}-\mathrm{h}}^{\mathrm{t}-\rho_{\mathrm{N}-1} \mathrm{~h}} \mathbf{x}^{\top}(\mathrm{s}) \mathbf{R}_{\mathrm{N}} \mathbf{x}(\mathrm{s}) \mathrm{d} s, \\
& \mathbf{V}_{3}\left(\mathbf{x}_{\mathbf{t}}\right)=\int_{t-\tau}^{t}(s-(t-\tau)) \dot{\mathbf{x}}^{\top}(s) \mathbf{W} \dot{\mathbf{x}}(s) d s+\int_{t-\rho_{1} h}^{t}\left(s-\left(t-\rho_{1} h\right)\right) \dot{\mathbf{x}}^{\top}(s) \mathbf{W}_{1} \dot{\mathbf{x}}(s) d s, \\
& \mathbf{V}_{4}\left(\mathbf{x}_{\mathrm{t}}\right)=\sum_{\mathrm{i}=1}^{\mathrm{N}-2} \int_{\mathrm{t}-\rho_{\mathrm{i}+1} \mathrm{~h}}^{\mathrm{t}-\rho_{\mathrm{i}} \mathrm{h}} \int_{\theta}^{\mathrm{t}} \dot{\mathbf{x}}^{\top}(\mathrm{s}) \mathbf{W}_{\mathrm{i}+1} \dot{\mathbf{x}}(\mathrm{s}) \mathrm{d} s \mathrm{~d} \theta+\int_{\mathrm{t}-\mathrm{h}}^{\mathrm{t}-\rho_{\mathrm{N}-1} \mathrm{~h}} \int_{\theta}^{\mathrm{t}} \dot{\mathbf{x}}^{\top}(\mathrm{s}) \mathbf{W}_{\mathrm{N}} \dot{\mathbf{x}}(\mathrm{s}) \mathrm{d} s \mathrm{~d} \theta, \\
& \mathbf{V}_{5}\left(\mathbf{x}_{\mathbf{t}}\right)=2 \sum_{s=1}^{m} g_{s} \int_{0}^{\sigma_{s}(t)} f_{s}(s) d s+2 \sum_{s=1}^{m} l_{s} \int_{0}^{\sigma_{s}(t)}\left[k_{s} s-f_{s}(s)\right] d s .
\end{aligned}
$$

Taking the derivative of $\mathbf{V}\left(\mathbf{x}_{\mathbf{t}}\right)$ along the trajectory of the NTLNCS (2.1), we can obtain the following differential:

$$
\dot{\mathbf{V}}\left(\mathbf{x}_{\mathrm{t}}\right)=\dot{\mathbf{V}}_{1}\left(\mathbf{x}_{\mathrm{t}}\right)+\dot{\mathbf{V}}_{2}\left(\mathbf{x}_{\mathrm{t}}\right)+\dot{\mathbf{V}}_{3}\left(\mathbf{x}_{\mathrm{t}}\right)+\dot{\mathbf{V}}_{4}\left(\mathbf{x}_{\mathrm{t}}\right)+\dot{\mathbf{V}}_{5}\left(\mathbf{x}_{\mathrm{t}}\right)
$$

where

$$
\begin{aligned}
\dot{\mathbf{V}}_{1}\left(\mathbf{x}_{t}\right)= & 2 \mathbf{x}^{\top}(t) P \dot{\mathbf{x}}(t)+\mathbf{x}^{\top}(t) \mathbf{P}_{1} \mathbf{x}(t)-(1-\dot{\tau}(t)) \mathbf{x}^{\top}(t-\tau(t)) \mathbf{P}_{1} \mathbf{x}(t-\tau(t)) \\
& +\dot{\mathbf{x}}^{\top}(t) \mathbf{P}_{2} \dot{\mathbf{x}}(t)-(1-\dot{\tau}(t)) \dot{\mathbf{x}}^{\top}(t-\tau(t)) P_{2} \dot{\mathbf{x}}(t-\tau(t)) \\
\leqslant & 2 \mathbf{x}^{\top}(t) P \dot{\mathbf{x}}(t)+\mathbf{x}^{\top}(t) \mathbf{P}_{1} \mathbf{x}(t)-\left(1-\tau_{c}\right) \mathbf{x}^{\top}(t-\tau(t)) \mathbf{x}_{1} \mathbf{x}(t-\tau(t)) \\
& +\dot{\mathbf{x}}^{\top}(t) \mathbf{x}_{2} \dot{\mathbf{x}}(t)-\left(1-\tau_{c}\right) \dot{\mathbf{x}}^{\top}(t-\tau(t)) \mathbf{P}_{2} \dot{\mathbf{x}}(t-\tau(t)) \\
= & \vartheta^{\top}(t)\left[\mathbf{e}_{1} \mathbf{P}_{1} \mathbf{e}_{1}^{\top}+\left\langle\mathbf{e}_{1} \mathbf{P e}_{2}^{\top}\right\rangle_{s}+\mathbf{e}_{2} \mathbf{P}_{2} \mathbf{e}_{2}^{\top}-\left(1-\tau_{c}\right) \mathbf{e}_{3} \mathbf{P}_{1} \mathbf{e}_{3}^{\top}-\left(1-\tau_{c}\right) \mathbf{e}_{4} \mathbf{P}_{2} \mathbf{e}_{4}^{\top}\right] \vartheta(t), \\
\dot{\mathbf{V}}_{2}\left(x_{t}\right)= & \mathbf{x}^{\top}(t) \mathbf{R}_{1} \mathbf{x}(t)-\mathbf{x}^{\top}\left(t-\rho_{1} h\right) \mathbf{R}_{1} \mathbf{x}\left(t-\rho_{1} h\right) \\
& +\sum_{i=1}^{N-2}\left[\mathbf{x}^{\top}\left(t-\rho_{i} h\right) \mathbf{R}_{i+1} \mathbf{x}\left(t-\rho_{i} h\right)-\mathbf{x}^{\top}\left(t-\rho_{i} h\right) \mathbf{R}_{i+1} \mathbf{x}\left(t-\rho_{i} h\right)\right]
\end{aligned}
$$




$$
\begin{aligned}
& +\mathbf{x}^{\top}\left(t-\rho_{N-1} h\right) \mathbf{R}_{N} \mathbf{x}\left(t-\rho_{N-1} h\right)-\mathbf{x}^{\top}(t-h) \mathbf{R}_{N} \mathbf{x}(t-h) \\
= & \mathbf{x}^{\top}(t) \mathbf{R}_{1} \mathbf{x}(t)-\mathbf{x}^{\top}(t-h) \mathbf{R}_{N} \mathbf{x}(t-h)+\sum_{i=1}^{N-1} \mathbf{x}^{\top}\left(t-\rho_{i} h\right)\left[\mathbf{R}_{i+1}-\mathbf{R}_{i}\right] \mathbf{x}\left(t-\rho_{i} h\right) \\
= & \vartheta^{\top}(t)\left[\mathbf{e}_{1} \mathbf{R}_{1} \mathbf{e}_{1}^{\top}-\mathbf{e}_{5+N} \mathbf{R}_{N} \mathbf{e}_{5+N}^{\top}+\sum_{i=1}^{N-1} \mathbf{e}_{i+5}\left[\mathbf{R}_{i+1}-\mathbf{R}_{i}\right] \mathbf{e}_{i+5}^{\top}\right] \vartheta(t) .
\end{aligned}
$$

From Lemma 2.1 and the celebrated Jensen's inequality [7], we can have

$$
\begin{aligned}
& \dot{\mathbf{V}}_{3}\left(\mathbf{x}_{\mathrm{t}}\right)=\dot{\mathbf{e}}^{\top}(\mathrm{t})\left(\tau \mathbf{W}+\rho_{1} \mathrm{~h} \mathbf{W}_{1}\right) \dot{\mathbf{x}}(\mathrm{t})-\int_{t-\tau}^{t} \dot{\mathbf{x}}^{\top}(s) \mathbf{W} \dot{\mathbf{x}}(s) \mathrm{d} s-\int_{t-\rho_{1} h}^{t} \dot{\mathbf{x}}^{\top}(s) W_{1} \dot{x}(s) d s \\
& \leqslant \dot{\mathbf{x}}^{\top}(\mathrm{t})\left(\tau \mathbf{W}+\rho_{1} \mathrm{~h} \mathbf{W}_{1}\right) \dot{\mathbf{x}}(t)-\int_{t-\tau(t)}^{t} \dot{\mathbf{x}}^{\top}(s) \mathbf{W} \dot{\mathbf{x}}(s) d s-\int_{t-\rho_{1} h}^{t} \dot{\mathbf{x}}^{\top}(s) \mathbf{W}_{1} \dot{\mathbf{x}}(s) d s \\
& \leqslant \dot{\mathbf{x}}^{\top}(\mathrm{t})\left(\tau \mathbf{W}+\rho_{1} h \mathbf{W}_{1}\right) \dot{\mathbf{x}}(\mathrm{t}) \\
& -\frac{1}{\tau}\left[\begin{array}{c}
\mathbf{x}(t) \\
\mathbf{x}(t-\tau(t))
\end{array}\right]^{\top}\left[\begin{array}{cc}
\mathbf{W} & -\mathbf{W} \\
-\mathbf{W} & \mathbf{W}
\end{array}\right]\left[\begin{array}{c}
\mathbf{x}(\mathbf{t}) \\
\mathbf{x}(\mathbf{t}-\tau(t))
\end{array}\right]\left[\begin{array}{c}
\mathbf{x}(\mathbf{t}) \\
\mathbf{x}(\mathbf{t}-\tau(t))
\end{array}\right] \\
& -\frac{1}{\rho_{1} h}\left[\begin{array}{c}
\mathbf{x}(t) \\
\mathbf{x}\left(t-\rho_{1} h\right) \\
\varpi_{1}(t)
\end{array}\right]^{\top}\left[\begin{array}{ccc}
4 \mathbf{W}_{1} & 2 \mathbf{W}_{1} & -6 \mathbf{W}_{1} \\
2 \mathbf{W}_{1} & 4 \mathbf{W}_{1} & -6 \mathbf{W}_{1} \\
-6 \mathbf{W}_{1} & -6 \mathbf{W}_{1} & 12 \mathbf{W}_{1}
\end{array}\right]\left[\begin{array}{c}
\mathbf{x}(\mathbf{t}) \\
\mathbf{x}\left(t-\rho_{1} h\right) \\
\varpi_{1}(t)
\end{array}\right] \\
& =\vartheta^{\top}(\mathbf{t})\left[\mathbf{e}_{2}\left(\tau \mathbf{W}+\rho_{1} h \mathbf{W}_{1}\right) \mathbf{e}_{2}^{\top}-\mathbf{e}_{1}\left(\frac{1}{\tau} \mathbf{W}+\frac{4}{\rho_{1} h} \mathbf{W}_{1}\right) \mathbf{e}_{1}^{\top}-\frac{1}{\tau} \mathbf{e}_{3} \mathbf{W} \mathbf{e}_{3}^{\top}-\frac{4}{\rho_{1} h} \mathbf{e}_{6} \mathbf{W}_{1} \mathbf{e}_{6}^{\top}\right. \\
& \left.-\frac{12}{\rho_{1} h} \mathbf{e}_{6+N} \mathbf{W}_{1} \mathbf{e}_{6+N}^{\top}+\left\langle\frac{1}{\tau} \mathbf{e}_{1} \mathbf{W} \mathbf{e}_{3}^{\top}-\frac{2}{\rho_{1} h} \mathbf{e}_{1} \mathbf{W}_{1} \mathbf{e}_{6}^{\top}+\frac{6}{\rho_{1} \mathrm{~h}} \mathbf{e}_{1} \mathbf{W}_{1} \mathbf{e}_{6+N}^{\top}+\frac{6}{\rho_{1} \mathrm{~h}} \mathbf{e}_{6} \mathbf{W}_{1} \mathbf{e}_{6+N}^{\top}\right\rangle_{s}\right] \vartheta(t), \\
& \dot{\mathbf{V}}_{4}\left(\mathbf{x}_{\mathrm{t}}\right)=\sum_{i=1}^{\mathrm{N}-1}\left(\rho_{i+1}-\rho_{i}\right) h \dot{\mathbf{x}}^{\top}(\mathrm{t}) \mathbf{W}_{\mathbf{i}+1} \dot{\mathbf{x}}(\mathrm{t})-\sum_{i=1}^{\mathrm{N}-1} \int_{t-\rho_{i+1} h}^{\mathrm{t}-\rho_{\mathrm{i}} \mathrm{h}} \dot{\mathbf{x}}^{\top}(\mathrm{s}) \mathbf{W}_{\boldsymbol{i}+1} \dot{\mathbf{x}}(\mathrm{s}) \mathrm{d} \mathrm{s} \\
& \leqslant \sum_{i=1}^{N-1}\left(\rho_{i+1}-\rho_{i}\right) h \dot{\mathbf{x}}^{\top}(t) \mathbf{W}_{\mathfrak{i}+1} \dot{\mathbf{x}}(t) \\
& -\sum_{i=1}^{N-1} \frac{1}{\left(\rho_{i+1}-\rho_{i}\right) h}\left[\begin{array}{c}
\mathbf{x}\left(t-\rho_{i} h\right) \\
\mathbf{x}\left(t-\rho_{i+1} h\right) \\
\varpi_{i+1}(t)
\end{array}\right]^{\top}\left[\begin{array}{ccc}
4 \mathbf{W}_{i+1} & 2 \mathbf{W}_{i+1} & -6 \mathbf{W}_{i+1} \\
2 \mathbf{W}_{i+1} & 4 \mathbf{W}_{i+1} & -6 \mathbf{W}_{i+1} \\
-6 \mathbf{W}_{i+1} & -6 \mathbf{W}_{i+1} & 12 \mathbf{W}_{i+1}
\end{array}\right]\left[\begin{array}{c}
\mathbf{x}\left(\mathbf{t}-\rho_{i} h\right) \\
\mathbf{x}\left(\mathbf{t}-\rho_{i+1} h\right) \\
\boldsymbol{\varpi}_{i+1}(\mathbf{t})
\end{array}\right] \\
& =\vartheta^{\top}(t)\left[\mathbf{e}_{2}\left(\sum_{i=1}^{N-1}\left(\rho_{i+1}-\rho_{i}\right) h \mathbf{W}_{i+1}\right) \mathbf{e}_{2}^{T}-\sum_{i=1}^{N-1} \frac{4}{\left(\rho_{i+1}-\rho_{i}\right) h} \mathbf{e}_{5+i} \mathbf{W}_{i+1} \mathbf{e}_{5+i}^{\top}\right. \\
& -\sum_{i=1}^{N-1} \frac{4}{\left(\rho_{i+1}-\rho_{i}\right) h} \mathbf{e}_{6+i} \mathbf{W}_{i+1} \mathbf{e}_{6+i}^{\top} \\
& -\sum_{i=1}^{N-1} \frac{12}{\left(\rho_{i+1}-\rho_{i}\right) h} \mathbf{e}_{6+N+i} \mathbf{W}_{i+1} \mathbf{e}_{6+N+i}^{T}+\left\langle\sum_{i=1}^{N-1} \frac{6}{\left(\rho_{i+1}-\rho_{i}\right) h} \mathbf{e}_{5+i} \mathbf{W}_{i+1} \mathbf{e}_{6+N+i}^{\top}\right. \\
& \left.\left.+\sum_{i=1}^{N-1} \frac{6}{\left(\rho_{i+1}-\rho_{i}\right) h} \mathbf{e}_{6+i} \mathbf{W}_{i+1} \mathbf{e}_{6+N+i}^{T}-\sum_{i=1}^{N-1} \frac{2}{\left(\rho_{i+1}-\rho_{i}\right) h} \mathbf{e}_{5+i} \mathbf{W}_{i+1} \mathbf{e}_{6+i}^{\top}\right\rangle_{s}\right] \vartheta(t), \\
& \dot{\mathbf{V}}_{5}\left(\mathbf{x}_{\mathrm{t}}\right)=2 \sum_{s=1}^{m}\left(g_{s}-l_{s}\right) \dot{\sigma}_{s}(t) f_{s}\left(\sigma_{s}(t)\right)+2 \sum_{s=1}^{m} \dot{\sigma}_{s}(t) l_{s} k_{s} \sigma_{s}(t) \\
& =2 \dot{\mathbf{x}}^{\top}(\mathrm{t}) \mathbf{H}(\mathbf{G}-\mathbf{L}) \mathbf{f}(\sigma(t))+2 \mathbf{x}^{\top}(\mathrm{t}) \mathbf{H} \mathbf{K L} \mathbf{H}^{\top} \dot{\mathbf{x}}(\mathrm{t}) \\
& =\vartheta^{\top}(t)\left\langle\mathbf{e}_{2} \mathbf{H}(G-\mathbf{L}) \mathbf{e}_{5}^{\top}+\mathbf{e}_{1} \mathbf{H K L H} \mathbf{H}^{\top} \mathbf{e}_{2}^{\top}\right\rangle_{s} \vartheta(t) \text {. }
\end{aligned}
$$


In order to improve the feasible region of stability criterion, according to (2.1), for any arbitrary matrices $\mathbf{T}_{1}, \mathbf{T}_{2}$ and $\mathbf{T}_{3}$ with appropriate dimensions, the following equation holds:

$$
\begin{aligned}
& \mathbf{0}=2\left[\dot{\mathbf{x}}^{\top}(\mathrm{t}) \mathbf{T}_{1}+\mathbf{x}^{\top}(\mathrm{t}) \mathbf{T}_{2}+\mathbf{f}^{\top}(\sigma(t)) \mathbf{T}_{3}\right]\left[-\dot{\mathbf{x}}(\mathrm{t})+\mathbf{A x}(\mathrm{t})+\mathbf{A}_{d} \mathbf{x}(t-h(t))+\mathbf{C} \dot{\mathbf{x}}(t-\tau(t))+\mathbf{D f}(\sigma(t))+\mathbf{N P}(t)\right] \\
& =\vartheta^{\top}(\mathbf{t})\left[\mathbf{e}_{1}\left\langle\mathbf{T}_{2} \mathbf{A}\right\rangle_{\mathrm{s}} \mathbf{e}_{1}^{\top}-\mathbf{e}_{2}\left\langle\mathbf{T}_{1}\right\rangle_{\mathrm{s}} \mathbf{e}_{2}^{\top}+\mathbf{e}_{5}\left\langle\mathbf{T}_{3} \mathbf{D}\right\rangle_{\mathrm{s}} \mathbf{e}_{5}^{\top}+\left\langle\mathbf{e}_{1}\left(\mathbf{A}^{\top} \mathbf{T}_{1}^{\top}-\mathbf{T}_{2}\right) \mathbf{e}_{2}^{\top}+\mathbf{e}_{2} \mathbf{T}_{1} \mathbf{A}_{\mathrm{d}} \mathbf{e}_{5+\mathrm{N}}^{\top}+\mathbf{e}_{2} \mathbf{T}_{1} \mathbf{C e}_{4}^{\top}+\mathbf{e}_{2}\left(\mathbf{T}_{1} \mathbf{D}\right.\right.\right. \\
& \left.-\mathbf{T}_{3}^{\top}\right) \mathbf{e}_{5}^{\top}+\mathbf{e}_{1} \mathbf{T}_{2} \mathbf{A}_{\mathrm{d}} \mathbf{e}_{5+\mathrm{N}}^{\mathrm{T}}+\mathbf{e}_{1} \mathbf{T}_{2} \mathbf{C} \mathbf{e}_{4}^{\top}+\mathbf{e}_{1}\left(\mathbf{T}_{2} \mathbf{D}+\mathbf{A}^{\mathrm{T}} \mathbf{T}_{3}^{\top}\right) \mathbf{e}_{5}^{\top}+\mathbf{e}_{5} \mathbf{T}_{3} \mathbf{A}_{\mathrm{d}} \mathbf{e}_{5+\mathrm{N}}^{\top}+\mathbf{e}_{4} \mathbf{C}^{\top} \mathbf{T}_{3}^{\top} \mathbf{e}_{5}^{\top} \\
& \left.\left.+\left(\mathbf{e}_{2} \mathbf{T}_{1}+\mathbf{e}_{1} \mathbf{T}_{2}+\mathbf{e}_{5} \mathbf{T}_{3}\right) \mathbf{N e}_{2 \mathrm{~N}+6}^{\top}\right\rangle_{s}\right] \vartheta(\mathrm{t}) \text {. }
\end{aligned}
$$

Moreover, the time-varying uncertain NTLNCS (2.1) with nonlinearity located in the sectors $\left[0, k_{s}\right]$, $(\mathrm{s}=1, \cdots, \mathrm{m})$, it is easy to achieve

$$
0<\frac{f_{s}\left(\sigma_{s}\right)}{\sigma_{s}} \leqslant k_{s}
$$

For any positive diagonal matrix $\mathbf{B}=\operatorname{diag}\left\{b_{1}, \cdots, b_{m}\right\}$, the following inequality holds:

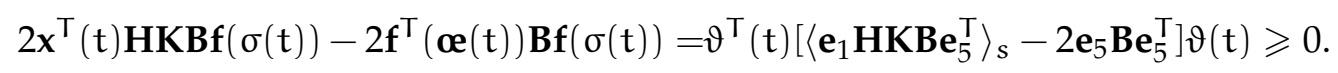

Furthermore, based on (2.4), for any positive scalar $\varepsilon$, the following inequality is satisfied

$$
\epsilon \vartheta^{\top}(t) \xi \vartheta^{\top} \xi \vartheta^{\top}(t)-\epsilon \mathbf{P}(t)^{\top} \mathbf{I P}(t)=\vartheta^{\top}(t)\left(\epsilon \xi^{\top} \xi-\epsilon \mathbf{e}_{2 N+6} \mathbf{I} \mathbf{e}_{2 N+6}^{\top}\right) \vartheta(t) \geqslant 0 .
$$

Combining Eqs. (3.3)-(3.12), it yields,

$$
\dot{\mathbf{V}}\left(\mathbf{x}_{\mathbf{t}}\right) \leqslant \vartheta^{\top}(\mathbf{t}) \Sigma \vartheta(\mathbf{t})
$$

From (3.1), we can have $\dot{\mathbf{V}}\left(t, x_{t}\right) \leqslant-\epsilon\|x(t)\|^{2}$ holds for any sufficiently small $\epsilon>0$. Therefore, this implies the NTLNCS (2.1) is globally asymptotically stable. The proof is completed.

Remark 3.2. In order to obtain less conservative stability criteria, the condition $0 \leqslant h(t) \leqslant h$ is divided into two equal subintervals such as $0 \leqslant h(t) \leqslant \frac{h}{2}$ and $\frac{h}{2} \leqslant h(t) \leqslant h$ in [5]. Different from the method [5], the condition $h_{L} \leqslant h(t) \leqslant h_{U}$ is divided into two unequal subintervals such as $h_{L} \leqslant h(t) \leqslant \alpha \frac{h_{L}+h_{U}}{2}$ and $\alpha \frac{h_{L}+h_{u}}{2} \leqslant h(t) \leqslant h_{U}$ by introducing an adjustable parameter $\alpha$ in [6], which can reduce further the conservatism of the existing results.

Remark 3.3. Stimulated by the existing approaches in $[5,6,19]$, we partition completely the time-varying delay interval $[0, h]$ into non-uniformly multiple subintervals $\sum_{i=1}^{N}\left[\rho_{i-1} h, \rho_{i} h\right]$ by bring in $N-1$ variable parameters $\rho_{i}$ satisfying $\left(0<\rho_{1}<\cdots<\rho_{i}<\cdots<\rho_{N-1}<1\right)$. It is worth pointing out that the method in $[5,19]$ is a special case of our method with $N=2$ and $\rho_{1}=\frac{1}{2}$ or $\rho_{i}=\frac{i}{N}(h(t)=h)$. Therefore, the proposed method in this paper is more general and less conservative.

Remark 3.4. Compared with those approaches in $[6,9,11,19,21]$ to deal with the integral term like $\int_{t-\rho_{i} h}^{t-\rho_{i-1} h} \dot{\mathbf{x}}^{\top}(s) \mathbf{W}_{i} \dot{\mathbf{x}}(s) d s$, Lemma 2.1 provides a new handling method. The method can establish the more relationship among

$$
\int_{t-\rho_{i} h}^{t-\rho_{i-1} h} \dot{\mathbf{x}}^{\top}(s) \mathbf{W}_{i} \dot{\mathbf{x}}(s) d s, \quad \frac{1}{\left(\rho_{i}-\rho_{i-1}\right) h} \int_{t-\rho_{i} h}^{t-\rho_{i-1} h} \mathbf{x}(s) d s, \quad \mathbf{x}\left(t-\rho_{i-1} h\right), \quad \text { and } \quad \mathbf{x}\left(t-\rho_{i} h\right),
$$

which may reduce further the conservatism of stability criteria.

Remark 3.5. If $\mathbf{C}=\mathbf{0}$, then the NTLNCS (2.1) reduces to the following delayed Lurie nonlinear control systems (DLNCS):

$$
\left\{\begin{array}{l}
\dot{\mathbf{x}}(\mathrm{t})=(\mathbf{A}+\triangle \mathbf{A}(\mathrm{t})) \mathbf{x}(\mathrm{t})+\left(\mathbf{A}_{\mathrm{d}}+\triangle \mathbf{A}_{\mathrm{d}}(\mathrm{t})\right) \mathbf{x}(\mathrm{t}-\mathrm{h})+(\mathbf{D}+\triangle \mathbf{D}(\mathrm{t})) \mathbf{f}(\sigma(\mathrm{t})), \\
\sigma(\mathrm{t})=\mathbf{H}^{\top} \mathbf{x}(\mathrm{t})=\left[\mathbf{h}_{1}, \cdots, \mathbf{h}_{\mathrm{m}}\right]^{\top} \mathbf{x}(\mathrm{t}), \quad \mathrm{t} \geqslant 0 \\
\mathbf{x}(\mathrm{t})=\phi(\mathrm{t}), \quad \mathrm{t} \in[-\max \{\mathrm{h}, \tau\}, 0] .
\end{array}\right.
$$

According to Theorem 3.1, we have the following corollary for the delay-dependent stability of DLNCS (3.14). 
Corollary 3.6. For given positive scalars $\mathrm{h}$ and $\varepsilon>0$, DLNCS (3.14) with nonlinearity located in the infinite sector $\mathrm{K}_{\left[0, \mathrm{k}_{s}\right]}$ is asymptotically stable if there exist positive definite matrices $\mathbf{P}>0, \mathbf{R}_{\mathbf{j}}>0, \mathbf{W}_{\mathbf{j}}>0(\mathbf{j}=$ $1, \cdots, \mathbf{N})$, any positive definite diagonal matrices $\mathbf{G}=\operatorname{diag}\left\{\mathrm{g}_{1}, \cdots, \mathrm{g}_{\mathrm{m}}\right\} \geqslant 0, \mathbf{L}=\operatorname{diag}\left\{\mathrm{l}_{1}, \cdots, \mathrm{l}_{\mathrm{m}}\right\} \geqslant 0$, $\mathbf{B}=\operatorname{diag}\left\{\mathrm{b}_{1}, \cdots, \mathrm{b}_{\mathrm{m}}\right\} \geqslant 0$, and arbitrary matrices $\mathbf{T}_{1}, \mathbf{T}_{2}$ and $\mathbf{T}_{3}$ with appropriate dimensions, such that the following LIM holds:

$$
\hat{\Sigma}<0
$$

where

$$
\begin{aligned}
& \hat{\Sigma}=\hat{\mathbf{e}}_{1}\left(\mathbf{R}_{1}-\frac{4}{\rho_{1} h} \mathbf{W}_{1}+\left\langle\mathbf{T}_{2} \mathbf{A}\right\rangle_{s}\right) \hat{\mathbf{e}}_{1}^{T}+\hat{\mathbf{e}}_{2}\left(\rho_{1} \mathrm{~h} \mathbf{W}_{1}+\sum_{i=1}^{\mathrm{N}-1}\left(\rho_{i+1}-\rho_{i}\right) h \mathbf{W}_{i+1}-\left\langle\mathbf{T}_{1}\right\rangle_{s}\right) \hat{\mathbf{e}}_{2}^{T}+\hat{\mathbf{e}}_{3}\left(\left\langle\mathbf{T}_{3} \mathbf{D}\right\rangle_{s}-2 \mathrm{~B}\right) \hat{\mathbf{e}}_{3}^{T} \\
& -\frac{4}{\rho_{1} \mathrm{~h}} \hat{\mathbf{e}}_{4} \mathbf{W}_{1} \hat{\mathbf{e}}_{4}^{\top}-\hat{\mathbf{e}}_{3+\mathrm{N}} \mathbf{R}_{\mathrm{N}} \hat{\mathbf{e}}_{3+\mathrm{N}}^{\top}-\frac{12}{\rho_{1} \mathrm{~h}} \hat{\mathbf{e}}_{4+\mathrm{N}} \mathbf{W}_{1} \hat{\mathbf{e}}_{4+\mathrm{N}}^{\top}+\left\langle\hat{\mathbf{e}}_{1}\left(\mathbf{P}+\mathbf{H} \mathbf{K L} \mathbf{H}^{\top}+\mathbf{A}^{\top} \mathbf{T}_{1}^{\top}-\mathbf{T}_{2}\right) \hat{\mathbf{e}}_{2}^{\top}+\hat{\mathbf{e}}_{2} \mathbf{T}_{1} \mathbf{A}_{\mathrm{d}} \hat{\mathbf{e}}_{3+\mathrm{N}}^{\top}\right. \\
& +\hat{\mathbf{e}}_{1}\left(\mathbf{T}_{2} \mathbf{D}+\mathbf{A}^{\mathrm{T}} \mathbf{T}_{3}^{\mathrm{T}}+\mathbf{H K B}\right) \hat{\mathbf{e}}_{3}^{\top}-\frac{2}{\rho_{1} \mathrm{~h}} \hat{\mathbf{e}}_{1} \mathbf{W}_{1} \hat{\mathbf{e}}_{4}^{\top}+\hat{\mathbf{e}}_{1} \mathbf{T}_{2} \mathbf{A}_{\mathrm{d}} \hat{\mathbf{e}}_{3+\mathrm{N}}^{\mathrm{T}} \\
& +\frac{6}{\rho_{1} h} \hat{\mathbf{e}}_{1} \mathbf{W}_{1} \hat{\mathbf{e}}_{4+\mathrm{N}}^{\top}+\hat{\mathbf{e}}_{2}\left(\mathbf{H}(\mathbf{G}-\mathbf{L})+\mathbf{T}_{1} \mathbf{D}-\mathbf{T}_{3}^{\top}\right) \hat{\mathbf{e}}_{3}^{\top} \\
& +\hat{\mathbf{e}}_{3} \mathbf{T}_{3} \mathbf{A}_{\mathrm{d}} \hat{\mathbf{e}}_{3+\mathrm{N}}^{\mathrm{T}}+\frac{6}{\rho_{1} \mathrm{~h}} \hat{\mathbf{e}}_{4} \mathbf{W}_{1} \hat{\mathbf{e}}_{4+\mathrm{N}}^{\mathrm{T}}+\sum_{\mathrm{i}=1}^{\mathrm{N}-1} \frac{6}{\left(\rho_{\mathrm{i}+1}-\rho_{\mathrm{i}}\right) \mathrm{h}} \hat{\mathbf{e}}_{3+\mathrm{i}} \mathbf{W}_{\mathrm{i}+1} \hat{\mathbf{e}}_{4+\mathrm{N}+\mathrm{i}}^{\mathrm{T}} \\
& +\sum_{i=1}^{N-1} \frac{6}{\left(\rho_{i+1}-\rho_{i}\right) h} \hat{\mathbf{e}}_{4+i} W_{i+1} \hat{\mathbf{e}}_{4+N+i}^{T} \\
& -\sum_{i=1}^{N-1} \frac{6}{\left(\rho_{i+1}-\rho_{i}\right) h} \hat{\mathbf{e}}_{3+i} W_{i+1} \hat{\mathbf{e}}_{4+i}^{\top}+\left(\hat{\mathbf{e}}_{2} \mathbf{T}_{1}+\left(\hat{\mathbf{e}}_{1} \mathbf{T}_{2}+\left(\hat{\mathbf{e}}_{3} \mathbf{T}_{3}\right) \mathbf{N}\left(\hat{\mathbf{e}}_{2 \mathrm{~N}+4}^{\top}\right)_{s}+\sum_{i=1}^{\mathrm{N}-1} \hat{\mathbf{e}}_{i+3}\left[\mathbf{R}_{i+1}-\mathbf{R}_{i}\right) \hat{\mathbf{e}}_{i+3}^{\top}\right.\right. \\
& -\sum_{i=1}^{N-1} \frac{4}{\left(\rho_{i+1}-\rho_{i}\right) h} \hat{\mathbf{e}}_{3+i} \mathbf{W}_{i+1} \hat{\mathbf{e}}_{3+i}^{\top}-\sum_{i=1}^{N-1} \frac{4}{\left(\rho_{i+1}-\rho_{i}\right) h} \hat{\mathbf{e}}_{4+i} \mathbf{W}_{i+1} \hat{\mathbf{e}}_{4+i}^{\top} \\
& -\sum_{i=1}^{\mathrm{N}-1} \frac{12}{\left(\rho_{i+1}-\rho_{i}\right) \mathrm{h}} \hat{\mathbf{e}}_{4+\mathrm{N}+\mathrm{i}} W_{i+1} \hat{\mathbf{e}}_{4+\mathrm{N}+\mathrm{i}}^{\mathrm{T}}+\epsilon \hat{\xi}^{\mathrm{T}} \hat{\xi}-\epsilon \hat{\mathbf{e}}_{2 \mathrm{~N}+4} \mathbf{I} \mathbf{e}_{2 \mathrm{~N}+4}^{\mathrm{T}}, \hat{\xi} \\
& =[\mathbf{E}_{1}, 0, \mathbf{E}_{3}, \overbrace{0, \cdots, 0}^{\mathrm{N}-1}, \mathbf{E}_{2}, \overbrace{0, \cdots, 0}^{\mathrm{N}}] .
\end{aligned}
$$

Proof. We choose the following LKF for DLCS (3.14):

$$
\mathbf{V}\left(x_{\mathrm{t}}\right)=\tilde{\mathbf{V}}_{1}\left(\mathbf{x}_{\mathrm{t}}\right)+\mathbf{V}_{2}\left(\mathbf{x}_{\mathrm{t}}\right)+\tilde{\mathbf{V}}_{3}+\mathbf{V}_{4}\left(\mathbf{x}_{\mathrm{t}}\right)+\mathbf{V}_{5}\left(\mathbf{x}_{\mathrm{t}}\right),
$$

where $\mathbf{V}_{2}\left(\mathbf{x}_{\mathbf{t}}\right), \mathbf{V}_{4}\left(\mathbf{x}_{\mathbf{t}}\right)$ and $\mathbf{V}_{5}\left(\mathbf{x}_{\mathrm{t}}\right)$ are defined in (3.2). Besides, $\tilde{\mathbf{V}}_{1}\left(\mathbf{x}_{\mathbf{t}}\right)$ and $\tilde{\mathbf{V}}_{3}\left(\mathbf{x}_{\mathbf{t}}\right)$ are also defined in (3.2) with $\mathbf{P}_{1}=\mathbf{P}_{2}=\mathbf{W}=\mathbf{0}$. Similarly to the proof of Theorem 3.1, the result follows immediately. This completes the proof.

Remark 3.7. When $\mathbf{D}=\triangle \mathbf{D}(\mathrm{t})=\mathbf{0}$ and $\tau(\mathrm{t})=\tau$, we can get one delay-dependent asymptotical stability criterion for neutral system (2.1). We will show the stability criterion for this case in Corollary 3.8.

Corollary 3.8. For given positive scalars $\tau, h$ and $\varepsilon>0, N T L C S(2.1)$ with $\mathbf{D}=\mathbf{0}$ and $\tau(\mathrm{t})=\tau$ is stable if there exist positive definite matrices $\mathbf{P}>0, \mathbf{W}>0, \mathbf{P}_{\mathfrak{i}}>0, \mathbf{R}_{\mathbf{j}}>0, \mathbf{W}_{\mathbf{j}}>0(\boldsymbol{i}=1,2 ; \mathbf{j}=1, \cdots, \mathbf{N})$, and arbitrary matrices $\mathbf{T}_{1}$ and $\mathbf{T}_{2}$ with appropriate dimensions, such that the following LIM holds:

$$
\tilde{\Sigma}<0,
$$

where

$$
\tilde{\Sigma}=\tilde{\mathbf{e}}_{1}\left(\mathbf{P}_{1}+\mathbf{R}_{1}-\frac{1}{\tau} \mathbf{W}-\frac{4}{\rho_{1} h} \mathbf{W}_{1}+\left\langle\mathbf{T}_{2} \mathbf{A}\right\rangle_{\mathrm{s}}\right) \tilde{\mathbf{e}}_{1}^{\top}+\tilde{\mathbf{e}}_{2}\left(\mathbf{P}_{2}+\tau \mathbf{W}+\rho_{1} h \mathbf{W}_{1}+\sum_{i=1}^{\mathrm{N}-1}\left(\rho_{i+1}-\rho_{i}\right) h \mathbf{W}_{i+1}\right.
$$




$$
\begin{aligned}
& \left.-\left\langle\mathbf{T}_{1}\right\rangle_{s}\right) \tilde{\mathbf{e}}_{2}^{T}-\tilde{\mathbf{e}}_{3}\left(\mathbf{P}_{1}+\frac{1}{\tau} \mathbf{W}\right) \tilde{\mathbf{e}}_{3}^{\top}-\tilde{\mathbf{e}}_{4} \mathbf{P}_{2} \tilde{\mathbf{e}}_{4}^{T}-\frac{4}{\rho_{1} h} \tilde{\mathbf{e}}_{5} \mathbf{W}_{1} \tilde{\mathbf{e}}_{5}^{\top}-\tilde{\mathbf{e}}_{4+\mathrm{N}} \mathbf{R}_{\mathrm{N}} \tilde{\mathbf{e}}_{4+\mathrm{N}}^{\top}-\frac{12}{\rho_{1} \mathrm{~h}} \tilde{\mathbf{e}}_{5+\mathrm{N}} \mathbf{W}_{1} \tilde{\mathbf{e}}_{5+\mathrm{N}}^{T} \\
& +\left\langle\tilde{\mathbf{e}}_{1}\left(\mathbf{P}+\mathbf{A}^{\top} \mathbf{T}_{1}^{\top}-\mathbf{T}_{2}\right) \tilde{\mathbf{e}}_{2}^{\top}+\tilde{\mathbf{e}}_{1} \frac{1}{\tau} \mathbf{W} \tilde{\mathbf{e}}_{3}^{\top}+\tilde{\mathbf{e}}_{1} \mathbf{T}_{2} \mathbf{C} \tilde{\mathbf{e}}_{4}^{\top}-\frac{2}{\rho_{1} h} \mathbf{e}_{1} \mathbf{W}_{1} \tilde{\mathbf{e}}_{5}^{\top}+\tilde{\mathbf{e}}_{1} \mathbf{T}_{2} \mathbf{A}_{d} \tilde{\mathbf{e}}_{4+\mathrm{N}}^{T}+\frac{6}{\rho_{1} h} \tilde{\mathbf{e}}_{1} \mathbf{W}_{1} \tilde{\mathbf{e}}_{5+\mathrm{N}}^{T}\right. \\
& +\tilde{\mathbf{e}}_{2} \mathbf{T}_{1} \mathbf{C} \tilde{\mathbf{e}}_{4}^{\top}+\tilde{\mathbf{e}}_{2} \mathbf{T}_{1} \mathbf{A}_{\mathrm{d}} \tilde{\mathbf{e}}_{4+\mathrm{N}}^{\top}+\frac{6}{\rho_{1} \mathrm{~h}} \tilde{\mathbf{e}}_{5} \mathbf{W}_{1} \tilde{\mathbf{e}}_{5+\mathrm{N}}^{\top}+\sum_{i=1}^{\mathrm{N}-1} \frac{6}{\left(\rho_{i+1}-\rho_{i}\right) \mathrm{h}} \tilde{\mathbf{e}}_{4+i} \mathbf{W}_{i+1} \tilde{\mathbf{e}}_{5+\mathrm{N}+\mathrm{i}}^{T} \\
& \left.\left.+\sum_{i=1}^{N-1} \frac{6}{\left(\rho_{i+1}-\rho_{i}\right) h} \tilde{\mathbf{e}}_{5+i} \mathbf{W}_{i+1} \tilde{\mathbf{e}}_{5+N+i}^{T}-\sum_{i=1}^{N-1} \frac{2}{\left(\rho_{i+1}-\rho_{i}\right) h} \tilde{\mathbf{e}}_{4+i} \mathbf{W}_{i+1} \tilde{\mathbf{e}}_{5+i}^{T}+\tilde{\mathbf{e}}_{2} \mathbf{T}_{1}+\tilde{\mathbf{e}}_{1} \mathbf{T}_{2}\right) \mathbf{N} \tilde{\mathbf{e}}_{2 \mathrm{~N}+5}^{T}\right\rangle_{s} \\
& +\sum_{i=1}^{N-1} \tilde{\mathbf{e}}_{i+4}\left(\mathbf{R}_{i+1}-\mathbf{R}_{i}\right) \tilde{\mathbf{e}}_{i+4}^{T}-\sum_{i=1}^{N-1} \frac{4}{\left(\rho_{i+1}-\rho_{i}\right) h} \tilde{\mathbf{e}}_{4+i} \mathbf{W}_{\mathfrak{i}+1} \tilde{\mathbf{e}}_{4+i}^{T}-\sum_{i=1}^{N-1} \frac{4}{\left(\rho_{i+1}-\rho_{i}\right) h} \tilde{\mathbf{e}}_{5+i} \mathbf{W}_{i+1} \tilde{\mathbf{e}}_{5+i}^{T} \\
& -\sum_{i=1}^{N-1} \frac{12}{\left(\rho_{i+1}-\rho_{i}\right) h} \tilde{\mathbf{e}}_{5+N+i} \mathbf{W}_{i+1} \tilde{\mathbf{e}}_{5+N+i}^{T}+\epsilon \tilde{\xi}^{\top} \tilde{\xi}-\epsilon \tilde{\mathbf{e}}_{2 N+4} \mathbf{I}_{2 N+4}^{T}, \tilde{\xi}=[\mathbf{E}_{1}, 0, \mathbf{E}_{3}, \overbrace{0, \cdots, 0}^{N-1}, \overbrace{0, \cdots, 0}^{N}] .
\end{aligned}
$$

Proof. We consider the following LKF candidate:

$$
\mathbf{V}\left(\mathbf{x}_{\mathrm{t}}\right)=\mathbf{V}_{1}\left(\mathbf{x}_{\mathrm{t}}\right)+\mathbf{V}_{2}\left(\mathbf{x}_{\mathrm{t}}\right)+\mathbf{V}_{3}\left(\mathbf{x}_{\mathrm{t}}\right)+\mathbf{V}_{4}\left(\mathbf{x}_{\mathrm{t}}\right)+\mathbf{V}_{5}\left(\mathbf{x}_{\mathrm{t}}\right),
$$

where

$$
\begin{aligned}
& \mathbf{V}_{1}\left(\mathbf{x}_{\mathrm{t}}\right)=\mathbf{x}^{\top}(\mathrm{t}) \mathbf{P x}(\mathrm{t}), \\
& \mathbf{V}_{2}\left(\mathbf{x}_{\mathbf{t}}\right)=\int_{t-\tau}^{t} \mathbf{x}^{\top}(s) \mathbf{P}_{1} \mathbf{x}(s) d s+\int_{t-\tau}^{t} \dot{\mathbf{x}}^{\top}(s) \mathbf{P}_{2} \dot{\mathbf{x}}(s) d s+\int_{t-\tau}^{t} \tau(s-(t-\tau)) \dot{\mathbf{x}}^{\top}(s) \mathbf{W} \dot{\mathbf{x}}(s) d s, \\
& \mathbf{V}_{3}\left(\mathbf{x}_{\mathbf{t}}\right)=\int_{t-\rho_{1} h}^{t} \mathbf{x}^{\top}(s) \mathbf{R}_{1} \mathbf{x}(s) \mathrm{d} s+\sum_{i=1}^{N-2} \int_{t-\rho_{i+1} h}^{t-\rho_{i} h} \mathbf{x}^{\top}(s) \mathbf{R}_{i+1} \mathbf{x}(s) d s+\int_{t-h}^{t-\rho_{N-1} h} \mathbf{x}^{\top}(s) \mathbf{R}_{N} \mathbf{x}(s) d s, \\
& \mathbf{V}_{4}\left(\mathbf{x}_{\mathbf{t}}\right)=\int_{t-\rho_{1} h}^{t} \rho_{1} h\left(s-\left(t-\rho_{1} h\right) \dot{\mathbf{x}}^{\top}(s) \mathbf{W}_{1} \dot{\mathbf{x}}(s) d s+\sum_{i=1}^{N-2} \int_{t-\rho_{i+1} h}^{t-\rho_{i} h} \int_{\theta}^{t}\left(\rho_{i+1} h-\rho_{i} h\right) \dot{\mathbf{x}}^{\top}(s) \mathbf{W}_{i+1} \dot{\mathbf{x}}(s) d s d \theta,\right. \\
& \mathbf{V}_{5}\left(\mathbf{x}_{\mathrm{t}}\right)=\int_{\mathrm{t}-\mathrm{h}}^{\mathrm{t}-\rho_{\mathrm{N}-1} \mathrm{~h}} \int_{0}^{\mathrm{t}}\left(\mathrm{h}-\rho_{\mathrm{N}-1} \mathrm{~h}\right) \dot{\mathbf{x}}^{\top}(\mathrm{s}) \mathbf{W}_{\mathrm{N}} \dot{\mathbf{x}}(\mathrm{s}) \mathrm{d} \mathrm{s} \mathrm{d} \theta .
\end{aligned}
$$

Then similar to the proof of Theorem 3.1, the result follows immediately. This completes the proof.

\section{Stability analysis in the infinite sector $[0, \infty]$}

Next, we consider the case in which the nonlinearities $f_{s}(\cdot)$ satisfy the infinite sector condition.

Theorem 4.1. For given positive scalars $\tau, \tau_{c}, h$ and $\varepsilon>0$, the NTLNCS (2.1) with nonlinearity located in the finite sector $\mathrm{K}_{[0, \infty]}$ is asymptotically stable if $\rho(\mathbf{C})<1$ and there exist positive definite matrices $\mathbf{P}>0, \mathbf{W}>0$, $\mathbf{P}_{\mathfrak{i}}>0, \mathbf{R}_{\mathbf{j}}>0, \mathbf{W}_{\mathbf{j}}>0(\boldsymbol{i}=1,2 ; \mathbf{j}=1, \cdots, \mathbf{N})$, a scalar $\alpha>0$ and any positive definite diagonal matrices $\mathbf{G}=\operatorname{diag}\left\{g_{1}, \cdots, g_{m}\right\} \geqslant 0$, and arbitrary matrices $\mathbf{T}_{1}, \mathbf{T}_{2}$ and $\mathbf{T}_{3}$ with appropriate dimensions, such that the following LIM holds:

$$
\check{\Sigma}+\check{\Xi}<0,
$$

where

$$
\check{\Sigma}=\Sigma-\left\langle\mathbf{e}_{1} \mathbf{H K L H}^{\top} \mathbf{e}_{2}^{\top}-\mathbf{e}_{2} \mathbf{H L e}_{5}^{\top}\right\rangle_{\mathrm{s}}, \quad \check{\Xi}=2 \alpha \mathbf{e}_{1} \mathbf{H e}_{5}^{\top} .
$$


Proof. When NTLCS (2.1) with nonlinearity located in the sector $[0, \infty]$, the condition (2.2) is equivalent to

$$
2 \alpha \mathbf{x}^{\top}(\mathrm{t}) \mathbf{H f}(\sigma(t))=\vartheta^{\top}(t)\left(2 \alpha \mathbf{e}_{1} \mathbf{H e}_{5}^{\top}\right) \vartheta(t)>0
$$

Then combining Eqs. (3.3)-(3.9), (3.11) and (4.2) yields,

$$
\dot{\mathbf{V}}\left(\mathbf{x}_{\mathbf{t}}\right) \leqslant \vartheta^{\top}(\mathbf{t})(\Sigma+\check{\Xi}) \vartheta(t), \quad j=1,2 .
$$

Then, similarly to the proof of Theorem 3.1, the result follows immediately. This completes the proof.

Remark 4.2. In many actual applications, maximum allowable time-delay upper bounds $h$ is of interested. Set $\alpha=\frac{1}{h}$, in Theorem 3.1 with fixed values $\tau_{c}$ and $\tau$, the optimal value can be obtained through the following optimization procedure:

$$
\left\{\begin{array}{l}
\text { Minize } \alpha \text { for } 0=\rho_{0}<\rho_{1}<\cdots<\rho_{i}<\cdots<\rho_{N-1}<\rho_{N}=1, \quad 0 \leqslant \delta \leqslant 1 \\
\text { Respect to }(3.1) .
\end{array}\right.
$$

Inequality (4.3) is a convex optimization problem and can be obtained efficiently by using the MATLABLMI Toolbox.

\section{Numerical examples}

In this section, five numerical simulation examples are given to show the effectiveness and correctness of the main results derived above.

Example 5.1. To demonstrate the superiority of our methods, we consider the following NTLNCS with the same parameters given in [9].

$$
\mathbf{A}=\left[\begin{array}{rr}
-2 & 0.5 \\
0.5 & -1
\end{array}\right], \quad \mathbf{A}_{\mathrm{d}}=\left[\begin{array}{cc}
1 & 0.4 \\
0.4 & -1
\end{array}\right], \quad \mathbf{C}=\left[\begin{array}{ll}
0.2 & 0.1 \\
0.1 & 0.2
\end{array}\right], \quad \mathbf{D}=\left[\begin{array}{c}
-0.5 \\
-0.75
\end{array}\right], \quad \mathbf{H}=\left[\begin{array}{l}
0.2 \\
0.6
\end{array}\right]
$$

Table 1: The maximum admissible upper of time delay $h$ for different values $\tau_{\mathrm{c}}$ in Example 5.1.

\begin{tabular}{c|c|c|c}
\hline$\tau_{c}$ & 0.1 & 0.5 & 0.9 \\
\hline$[9]$ & 4.7407 & 3.0562 & 0.1198 \\
\hline Theorem 4.1 $(\mathrm{N}=3)$ & 7.9511 & 4.7791 & 0.1223 \\
\hline Theorem 4.1 $(\mathrm{N}=4)$ & 8.1065 & 4.7886 & 0.1224 \\
\hline
\end{tabular}

For different $\tau_{\mathfrak{c}}$, by applying Theorem 4.1 and solving the LMI (4.1) via MATLAB LMI Control Toolbox, we can obtain the maximum allowable upper bounds $h=\tau$ which are listed in Table 1 . From Table 1, it can show crisply that our results are less conservative than the existing results in [9]. Therefore, it can be deserved that our method is more effective than those in [9].

Let $N=4, \rho_{1}=0.25, \rho_{2}=0.50, \rho_{3}=0.75, \tau_{c}=0.1$ and $h=\tau=8.1065$, we can acquire the following feasible parameters by Theorem 4.1 in our paper. Due to the limitation of the length of this paper, we only provide a part of the feasible solutions here

$$
\begin{aligned}
& \mathbf{P}=10^{4} *\left[\begin{array}{cc}
6.4957 & -1.51328 \\
-1.5132 & 9.3654
\end{array}\right], \quad \mathbf{R}_{1}=10^{5} *\left[\begin{array}{cc}
0.5344 & -0.2728 \\
-0.2728 & 1.0074
\end{array}\right], \quad \mathbf{R}_{2}=10^{5} *\left[\begin{array}{cc}
0.5386 & -0.2791 \\
-0.2791 & 1.0168
\end{array}\right], \\
& \mathbf{R}_{3}=10^{5} *\left[\begin{array}{cc}
0.5428 & -0.2854 \\
-0.2854 & 1.0261
\end{array}\right], \quad \mathbf{R}_{4}=10^{5} *\left[\begin{array}{cc}
0.5470 & -0.2916 \\
-0.2916 & 1.0355
\end{array}\right], \quad \mathbf{W}_{1}=10^{3} *\left[\begin{array}{cc}
1.7686 & -2.6284 \\
-2.6284 & 3.9099
\end{array}\right], \\
& \mathbf{W}_{2}=10^{3} *\left[\begin{array}{cc}
1.7673 & -2.6274 \\
-2.6274 & 3.9098
\end{array}\right], \quad \mathbf{W}_{3}=10^{3} *\left[\begin{array}{cc}
1.7668 & -2.6269 \\
-2.6269 & 3.9097
\end{array}\right], \quad \mathbf{W}_{4}=10^{3} *\left[\begin{array}{cc}
1.7665 & -2.6267 \\
-2.6267 & 3.9098
\end{array}\right],
\end{aligned}
$$




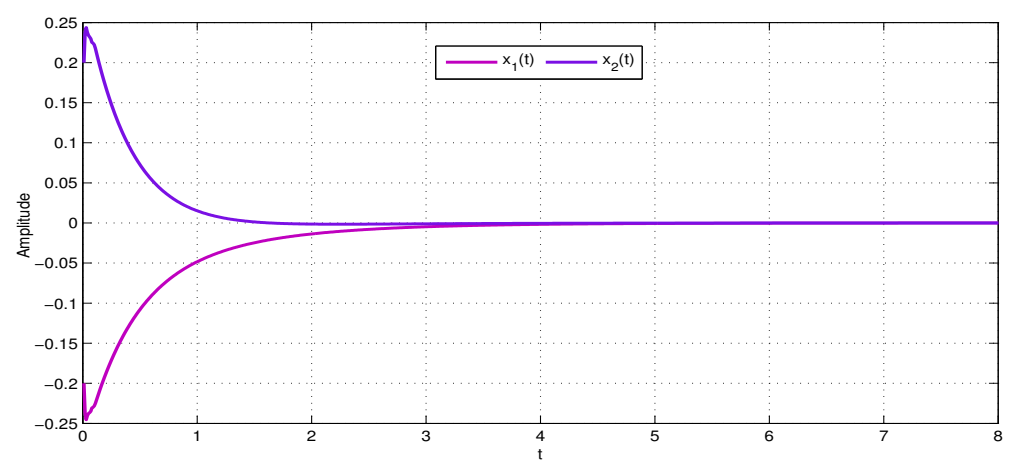

Figure 1: State trajectory of $\mathbf{x}(t)$ in the plane for $h=7.9511$ in Example 5.1.

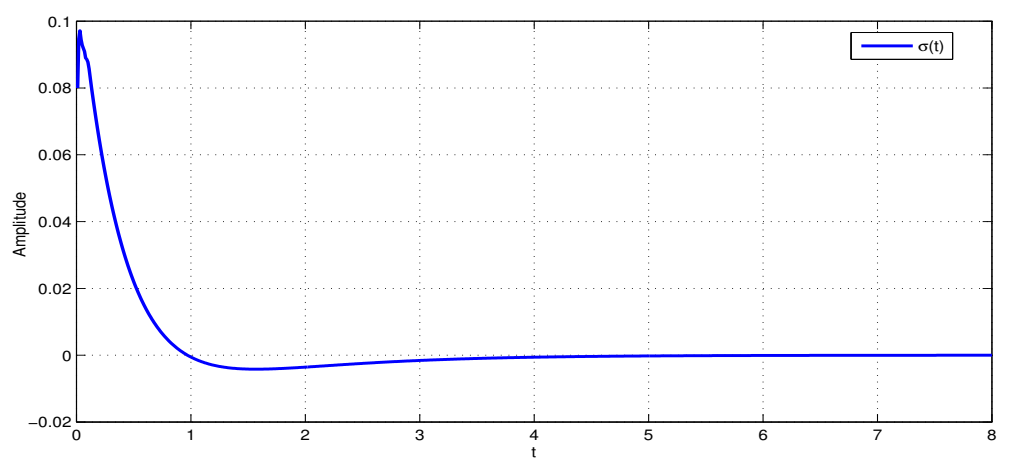

Figure 2: State trajectory of the output vector $\sigma(t)$ in the plane for $h=7.9511$ in Example 5.1.

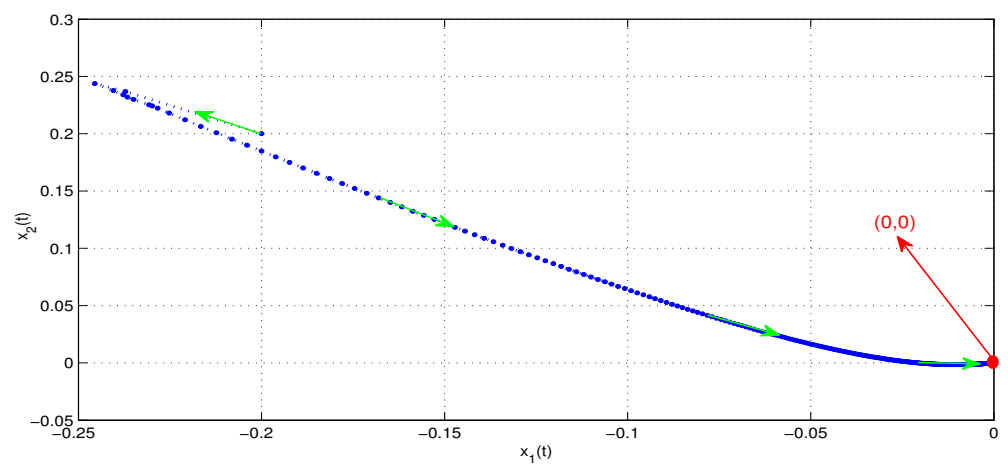

Figure 3: Phase trajectory of $\mathbf{x}(t)$ in the plane for $h=7.9511$ in Example 5.1.

Assume $\mathrm{N}=3, \rho_{1}=0.3, \rho_{2}=0.6$ and $\tau_{\mathrm{c}}=0.1$, by solving LMI (4.1) of Theorem 4.1, the maximum bound $h=\tau=7.9511$ is obtained. Besides, the simulation results with initial state $[-0.2,0.2]$ are given in Figures 1-3, which show that the state trajectories approach to zero asymptotically.

Example 5.2. Consider the following NTLNCS with the following parameters:

$$
\begin{array}{ll}
\mathbf{A}=\left[\begin{array}{cc}
-1.3 & 0.2 \\
0.1 & -1.2
\end{array}\right], \quad \mathbf{A}_{\mathrm{d}}=\left[\begin{array}{cc}
-1.1 & -0.2 \\
-0.1 & -1.1
\end{array}\right], \quad \mathbf{C}=\left[\begin{array}{cc}
-0.2 & 0 \\
0.2 & -0.1
\end{array}\right], \\
\mathbf{D}=\left[\begin{array}{cc}
-0.2 & 0.1 \\
-0.45 & -0.3
\end{array}\right], \quad \mathbf{H}=\left[\begin{array}{cc}
0.3 & -0.2 \\
0.3 & 0.1
\end{array}\right] .
\end{array}
$$


Table 2: The maximum admissible upper of time delay $\tau=\mathrm{h}$ for different values of $\mathbf{K}$ in Example 5.2.

\begin{tabular}{c|c|c|c}
\hline Method & Theorem 3.1 $\left(\mathbf{f}(\sigma(t)) \in \mathbf{K}_{1}\right)$ & Theorem 3.1 $\left(\mathbf{f}(\sigma(t)) \in \mathbf{K}_{2}\right)$ & Theorem 3.1 $\left(\mathbf{f}(\sigma(\mathbf{t})) \in \mathbf{K}_{3}\right)$ \\
\hline$\tau_{c}=0.1$ & 2.3218 & 1.6806 & 0.2769 \\
\hline
\end{tabular}

Let $\mathrm{N}=3, \rho_{1}=0.3, \rho_{2}=0.6$ and $\tau_{\mathrm{c}}=0.1$, the admissible upper bounds of $\tau=\mathrm{h}$ which guarantee asymptotically stable of the NTLCS (2.1) by applying Theorem 3.1 are listed in Table 2, where $\mathbf{K}_{1}=$ $\mathbf{K}[0,0.5), \mathbf{K}_{2}=\mathbf{K}[0,10), \mathbf{K}_{3}=\mathbf{K}[0,1000)$. For example, if $\mathbf{f}(\sigma(t)) \in \mathbf{K}_{2}=[0,0.5]$, then $\boldsymbol{h}=\tau=2.3218$, we can offer the following part feasible parameters by applying Theorem 3.1 in our paper:

$$
\begin{aligned}
& \mathbf{P}=\left[\begin{array}{cc}
12.0799 & -4.1587 \\
-4.1587 & 12.2488
\end{array}\right], \quad \mathbf{W}=\left[\begin{array}{cc}
0.4804 & -0.3098 \\
-0.3098 & 0.6440
\end{array}\right], \quad \mathbf{P}_{1}=\left[\begin{array}{cc}
2.4637 & -1.9127 \\
-1.9127 & 2.2126
\end{array}\right], \\
& \mathbf{P}_{2}=\left[\begin{array}{cc}
3.8392 & -1.7500 \\
-1.7500 & 2.6918
\end{array}\right], \quad \mathbf{R}_{1}=\left[\begin{array}{cc}
13.5852 & -3.6702 \\
-3.6702 & 13.0039
\end{array}\right], \quad \mathbf{R}_{2}=\left[\begin{array}{cc}
13.5286 & -2.8083 \\
-2.8083 & 13.2571
\end{array}\right], \\
& \mathbf{R}_{3}=\left[\begin{array}{cc}
13.5984 & -1.9204 \\
-1.9204 & 13.6192
\end{array}\right], \quad \mathbf{W}_{1}=\left[\begin{array}{ll}
1.3586 & 0.2622 \\
0.2622 & 1.6729
\end{array}\right], \quad \mathbf{W}_{2}=\left[\begin{array}{ll}
1.3715 & 0.2592 \\
0.2592 & 1.6700
\end{array}\right] .
\end{aligned}
$$

Moreover, when $h=2.3218, \mathbf{f}(\sigma(t))=(0.25+0.25 \operatorname{sign}(\sigma(t))) \sigma(t), k_{1}=0.5, k_{2}=0.5$, and $x(0)=[-1.5,1.8]$, the trajectories of variables $\mathbf{x}(\mathrm{t})$ and $\sigma(\mathrm{t})$ are shown in Figures 4-6.

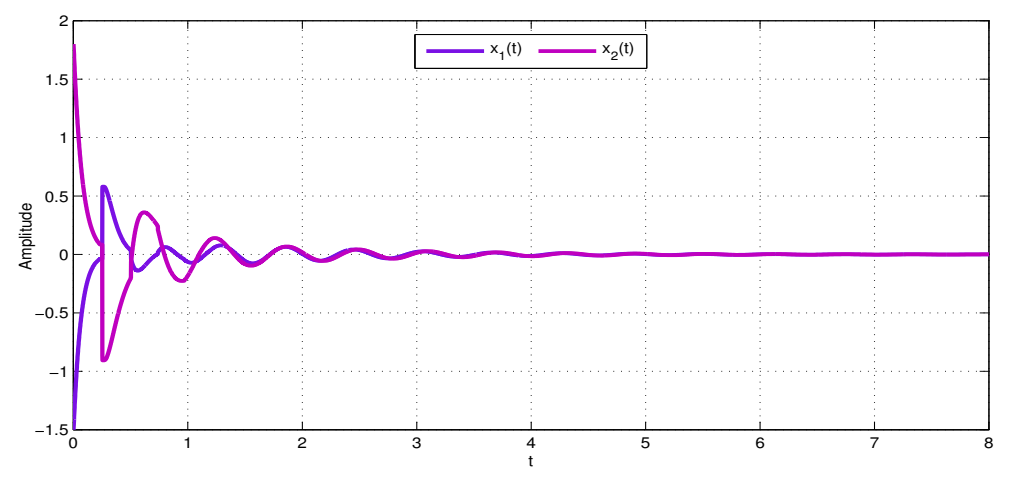

Figure 4: State trajectory of $\mathbf{x}(t)$ in the plane for $h=\tau=2.3218$ in Example 5.2.

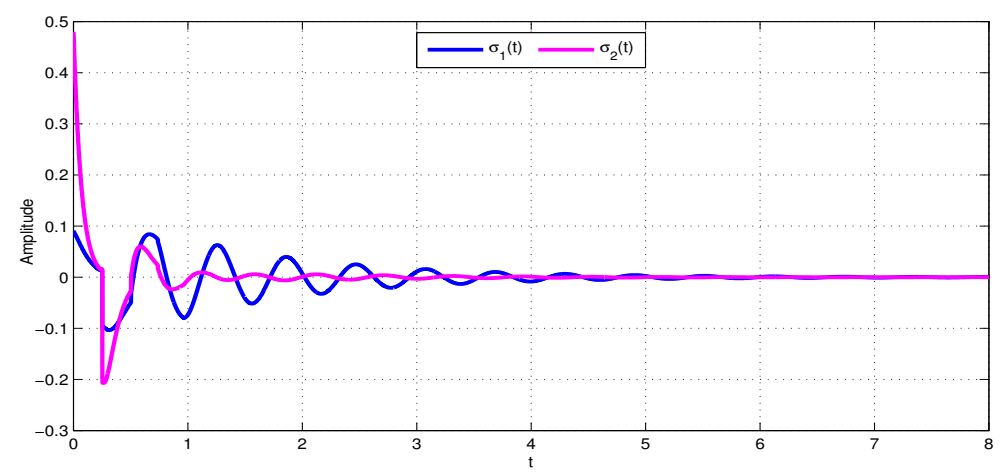

Figure 5: State trajectory of the output vector $\sigma(t)$ in the plane for $h=\tau=2.3218$ in Example 5.2. 


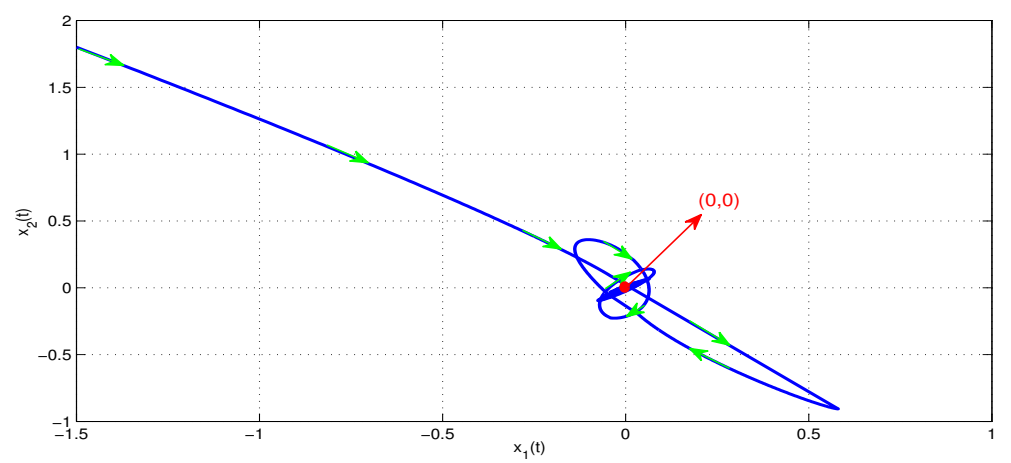

Figure 6: Phase trajectory of $\mathbf{x}(t)$ in the plane for $h=\tau=2.3218$ in Example 5.2.

Example 5.3. Consider the following Chua's circuit example and the system equation in $[5,11,21]$ which is given by

$$
\begin{aligned}
& \dot{\mathbf{x}}(\mathrm{t})=\alpha(\mathbf{y}(\mathrm{t})-\mathbf{h}(\mathrm{t})), \\
& \dot{\mathbf{y}}(\mathrm{t})=\mathbf{x}(\mathrm{t})-\mathbf{y}(\mathrm{t})+\mathbf{z}(\mathrm{t}), \\
& \dot{\mathbf{z}}(\mathrm{t})=-\beta \mathbf{y}(\mathrm{t}),
\end{aligned}
$$

with nonlinear characteristic

$$
h(\mathbf{x}(t))=m_{1} \mathbf{x}(t)+\frac{1}{2}\left(m_{0}-m_{1}\right)[|\mathbf{x}(t)+c|-|\mathbf{x}(t)-c|],
$$

and parameters $m_{0}=-\frac{1}{7}, m_{1}=\frac{2}{7}, \alpha=9, \beta=14.28$, and $c=1$. The system can be presented in normal LCS framework (3.14) with

$$
\mathbf{A}=\left[\begin{array}{ccc}
-\alpha \mathrm{m}_{1}-1 & \alpha & 0 \\
1 & -2 & 1 \\
0 & -\beta & -1
\end{array}\right], \quad \mathbf{A}_{\mathrm{d}}=\left[\begin{array}{ccc}
-6.0026 & 0 & 0 \\
-1.3367 & 0 & 0 \\
2.1264 & 0 & 0
\end{array}\right], \quad \mathbf{D}=\left[\begin{array}{c}
-\alpha\left(\mathrm{m}_{0}-\mathrm{m}_{1}\right) \\
0 \\
0
\end{array}\right], \quad \mathbf{H}=\left[\begin{array}{l}
1 \\
0 \\
0
\end{array}\right]
$$

The feedback nonlinear function belongs to the sector $[0,1]$.

Table 3: The maximum admissible upper of time delay $\mathrm{h}$ in Example 5.3.

\begin{tabular}{c|c|c|c|c|c}
\hline Method & {$[11]$} & {$[21]$} & {$[5]$} & Corollary 3.6 $(\mathrm{N}=3)$ & Corollary 3.6 $(\mathrm{N}=4)$ \\
\hline $\mathrm{h}$ & 0.1622 & 0.1745 & 0.1747 & 0.1785 & 0.1803 \\
\hline
\end{tabular}

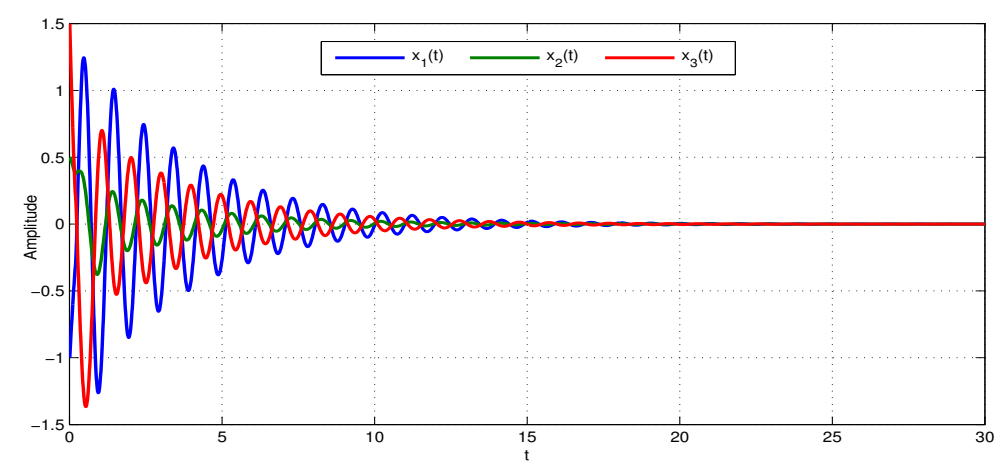

Figure 7: State trajectory of $\mathbf{x}(t)$ in the plane for $h=0.1785$ in Example 5.3. 


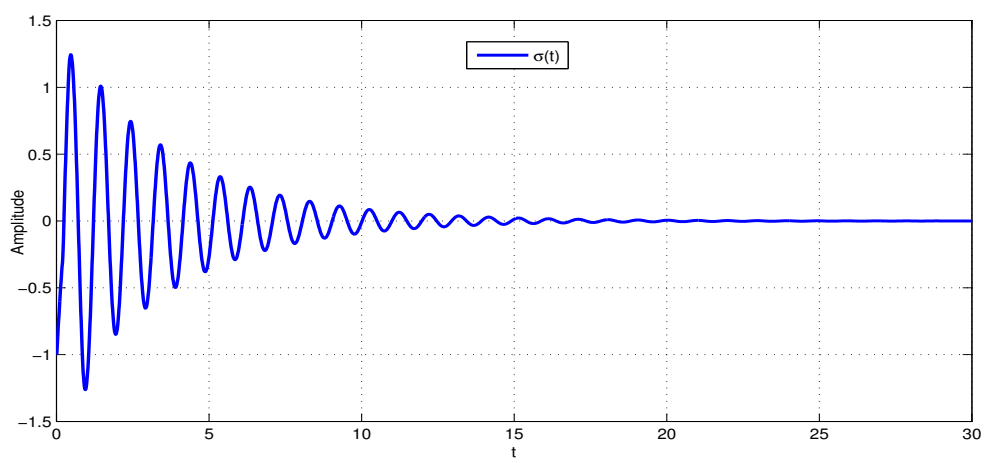

Figure 8: State trajectory of the output vector $\sigma(t)$ in the plane for $h=0.1785$ in Example 5.3.

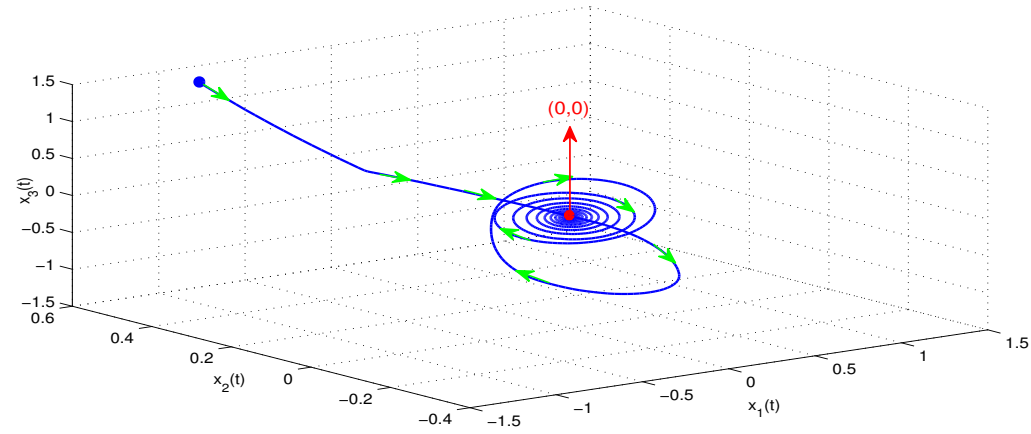

Figure 9: Phase trajectory of $\mathbf{x}(\mathrm{t})$ in the plane for $\mathrm{h}=0.1785$ in Example 5.3.

The maximum admissible upper of time delay $h$ for different $N$ by using Corollary 3.6 against the existing results in $[5,11,21]$ are listed in Table 3. From Table 3, it can be observed that the proposed stability criterion is less conservative than the ones in $[5,11,21]$, which does well out of using a general and complete DPM. On the other hand, under $x(0)=[-1,0.5,1.5]$, Figures 7-9 are the graphical simulations to show the states of $\mathbf{x}(\mathrm{t})$.

Example 5.4. Consider the following NTDS with parameters listed in $[13,17,18,39]$ and demonstrate the results of comparison between the existing methods and our method. Parameters are given as:

$$
\mathbf{A}=\left[\begin{array}{cc}
-0.9 & 0.2 \\
0.1 & -0.9
\end{array}\right], \quad \mathbf{A}_{\mathrm{d}}=\left[\begin{array}{cc}
-1.1 & -0.2 \\
-0.1 & -1.1
\end{array}\right], \quad \mathbf{C}=\left[\begin{array}{cc}
-0.2 & 0 \\
0.2 & -0.1
\end{array}\right]
$$

Table 4: The maximum admissible upper of time delay $\mathrm{h}$ for Example 5.4.

\begin{tabular}{c|c|c|c|c|c|c}
\hline Method & {$[39]$} & {$[13]$} & {$[17]$} & {$[18]$} & Corollary 3.8 $(\mathrm{N}=3)$ & Corollary 3.8 $(\mathrm{N}=4)$ \\
\hline $\mathrm{h}$ & 1.7856 & 1.8266 & 1.9132 & 2.0054 & 2.0649 & 2.0863 \\
\hline
\end{tabular}

When $\mathrm{N}=3$ and $\mathrm{N}=4$, the corresponding maximum admissible upper bounds derived by Corollary 3.8 is given in Table 4. From Table 4, it is clear to see that the stability criterion proposed in this paper gives much less conservative results than those in the existing literature $[13,17,18,39]$. Set $\rho_{1}=\frac{1}{3}$ and $\rho_{2}=\frac{2}{3}$, then $h=\tau=2.0649$, we can obtain the following feasible parameters by Corollary 3.8 in our paper:

$$
\mathbf{P}=\left[\begin{array}{cc}
249.4705 & 95.9433 \\
95.9433 & 469.1108
\end{array}\right], \quad \mathbf{P}_{1}=\left[\begin{array}{cc}
1.7884 & -1.7663 \\
-1.7663 & 3.3732
\end{array}\right], \quad \mathbf{P}_{2}=\left[\begin{array}{cc}
67.9874 & -57.0689 \\
-57.0689 & 99.0575
\end{array}\right],
$$




$$
\begin{aligned}
& \mathbf{W}=\left[\begin{array}{cc}
0.5049 & -0.4571 \\
-0.4571 & 0.9544
\end{array}\right], \quad \mathbf{R}_{1}=\left[\begin{array}{cc}
239.8302 & 64.7760 \\
64.7760 & 449.0936
\end{array}\right], \quad \mathbf{R}_{2}=\left[\begin{array}{ll}
291.8865 & 114.6423 \\
114.6423 & 555.4165
\end{array}\right], \\
& \mathbf{R}_{3}=\left[\begin{array}{ll}
343.8066 & 164.5078 \\
164.5078 & 661.8171
\end{array}\right], \quad \mathbf{W}_{1}=\left[\begin{array}{cc}
69.6059 & 67.2373 \\
67.2373 & 141.4748
\end{array}\right], \quad \mathbf{W}_{2}=\left[\begin{array}{cc}
69.5574 & 67.1758 \\
67.1758 & 141.5071
\end{array}\right], \\
& \mathbf{W}_{3}=\left[\begin{array}{cc}
69.3951 & 67.3458 \\
67.3458 & 141.6744
\end{array}\right], \quad \mathbf{T}_{1}=\left[\begin{array}{cc}
213.2610 & 80.6756 \\
79.8984 & 394.1250
\end{array}\right], \quad \mathbf{T}_{2}=\left[\begin{array}{cc}
248.9866 & 96.6918 \\
95.9811 & 468.0007
\end{array}\right] \text {. }
\end{aligned}
$$

Moreover, Figures 10 and 11 may show that the trajectories of NTDS are asymptotically stable. Similarly,

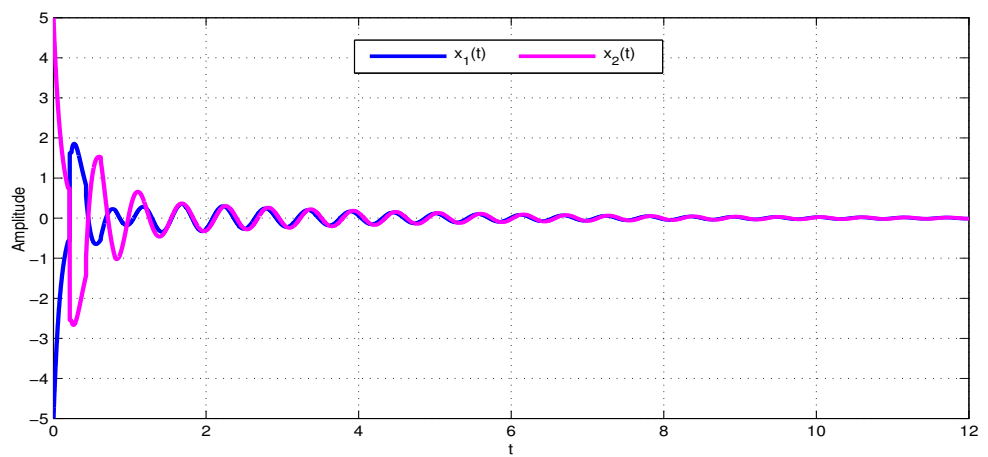

Figure 10: The response of the state dynamics $\mathbf{x}(t)$ in the plane for $h=2.0649$ in Example 5.4.

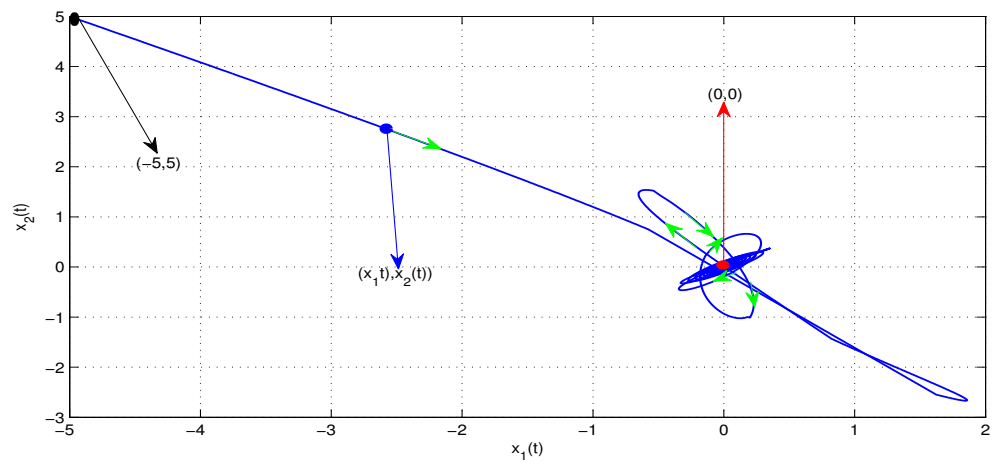

Figure 11: Phase trajectory of model of $\mathbf{x}(\mathrm{t})$ in the plane for $\mathrm{h}=2.0649$ in Example 5.4.

when $\mathrm{N}=4, \rho_{1}=0.25, \rho_{2}=0.50$ and $\rho_{3}=0.75$, we can obtain the maximum upper bound on the allowable delay to be $h=\tau=2.0863$.

Example 5.5. Finally, we consider the following NTDS with parameters listed in $[8,10,12,27]$ and demonstrate the results of comparison between the existing methods and our method. Parameters are given as:

$$
\mathbf{A}=\left[\begin{array}{cc}
-2 & 0 \\
0 & -0.9
\end{array}\right], \quad \mathbf{A}_{\mathrm{d}}=\left[\begin{array}{cc}
-11 & 0 \\
-1 & -1
\end{array}\right], \quad \mathbf{C}=\left[\begin{array}{cc}
0.1 & 0 \\
0 & 0.1
\end{array}\right]
$$

Table 5: The maximum admissible upper of time delay $\mathrm{h}$ for Example 5.5.

\begin{tabular}{c|c|c|c|c|c|c}
\hline Method & {$[8]$} & {$[10]$} & {$[27]$} & {$[12]$} & Corollary 3.8 $(\mathrm{N}=3)$ & Corollary 3.8 $(\mathrm{N}=4)$ \\
\hline $\mathrm{h}$ & 3.49 & 4.33 & 4.35 & 4.42 & 4.5262 & 4.5586 \\
\hline
\end{tabular}




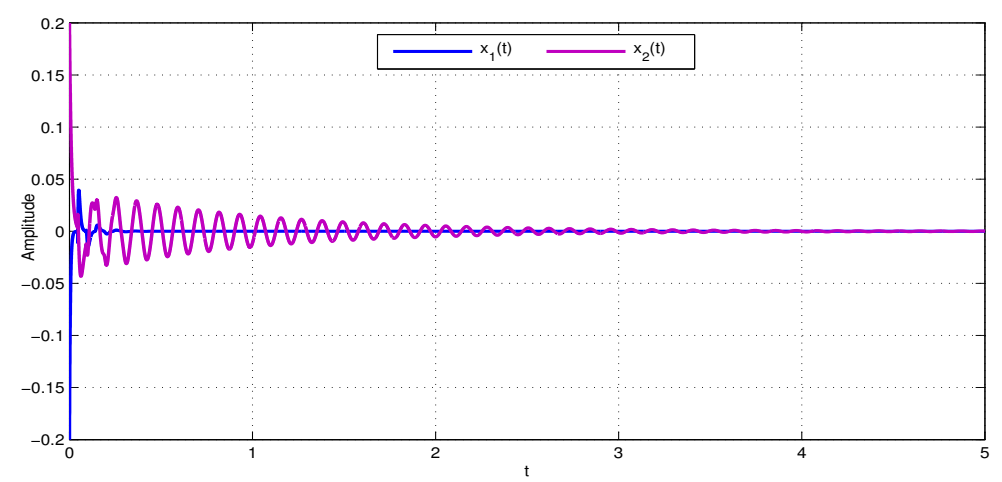

Figure 12: The response of the state dynamics $\mathbf{x}(\mathrm{t})$ in the plane for $\mathrm{h}=4.5262$ in Example 5.5.

For $\mathrm{N}=3, \rho_{1}=\frac{1}{3}$ and $\rho_{2}=\frac{2}{3}$, and $\mathrm{N}=4, \rho_{1}=\frac{1}{4}, \rho_{2}=\frac{1}{2}$ and $\rho_{2}=\frac{3}{4}$, the corresponding results for the maximum allowable upper bounds of the time delay $h=\tau$ are given in Table 5. From Table 5, we can see clearly that stability criterion established in this paper is less conservative than the results compared in $[8,10,12,27]$. Thus, it can be seen that WII used in this paper plays a key role reducing the conservatism of results. Besides, $\mathrm{N}=3$ and $\mathrm{h}=4.5262$, the response of the state trajectories for the above TDS which is asymptotically stable are shown in Figure 12.

\section{Conclusions}

In this paper, the problem of delay-dependent robust stability analysis for time-varying uncertain NTLNCS with mixed time delays is investigated by developing some new methods. In order to estimate the bounds of integral terms more exactly, we construct an augmented LKF based on DPM. Moreover, by getting the utmost out of WII, less conservative delay-dependent stability conditions are obtained in terms of LIMs. Finally, the feasibility and effectiveness of the proposed methods have been demonstrated by five numerical simulation examples. The foregoing results have the potential to be useful for the further study of TDSs.

\section{Acknowledgment}

This work was supported by National Basic Research Program of China (2010CB732501), National Natural Science Foundation of China (61273244, 61273015, 11526149,61603272,11601474 and 11461082), National Basic Research Program of China (Grant No. 61503064 and 51502338), the Youth Fund Project of Tianjin Natural Science Foundation (Grant No.16JCQNJC03900).

\section{References}

[1] P. Balasubramaniam, R. Krishnasamy, R. Rakkiyappan, Delay-dependent stability of neutral systems with time-varying delays using delay-decomposition approach, Appl. Math. Model., 36 (2012), 2253-2261. 1

[2] J.-W. Cao, Improved delay-dependent exponential stability criteria for time-delay system, J. Franklin Inst., 350 (2013), 790-801. 1

[3] W.-H. Chen, Z.-P. Wang, X.-M. Lu, On sampled-data control for master-slave synchronization of chaotic Lur'e systems, IEEE Trans. Circuits Syst. II, Exp. Briefs, 59 (2012), 515-519. 1

[4] Y.-C. Ding, H. Liu, J. Cheng, $\mathrm{H}_{\infty}$ filtering for a class of discrete-time singular Markovian jump systems with time-varying delays, ISA Trans., 53 (2014), 1054-1060. 1

[5] W.-Y. Duan, B.-Z. Du, Z.-F. Liu, Y. Zou, Improved stability criteria for uncertain neutral-type Lur'e systems with timevarying delays, J. Franklin Inst., 351 (2014), 4538-4554. 1, 3.2, 3.3, 5.3, 3, 5.3 
[6] W.-Y. Duan, B.-Z. Du, J. You, Y. Zou, Improved robust stability criteria for a class of Lur'e systems with interval timevarying delays and sector-bounded nonlinearity, Internat. J. Systems Sci., 46 (2015), 944-954. 1, 3.2, 3.3, 3.4

[7] M. Fang, J. H. Park, A multiple integral approach to stability of neutral time-delay systems, Appl. Math. Comput., 224 (2013), 714-718. 1, 3

[8] E. Fridman, U. Shaked, Delay-dependent stability and $\mathrm{H}_{\infty}$ control: constant and time-varying delays, Internat. J. Control, 76 (2003), 48-60. 1, 5.5, 5, 5.5

[9] J.-F. Gao, H.-Y. Su, X.-F. Ji, J. Chu, Stability analysis for a class of neutral systems with mixed delays and sector-bounded nonlinearity, Nonlinear Anal. Real World Appl., 9 (2008), 2350-2360. 1, 3.4, 5.1, 1, 5.1

[10] Q.-L. Han, Robust stability of uncertain delay-differential systems of neutral type, Automatica J. IFAC, 38 (2000), 719 723. $1,5.5,5,5.5$

[11] Q.-L. Han, On designing time-varying delay feedback controllers for master-slave synchronization of Lure systems, IEEE Trans. Circuits Syst. I. Regul. Pap., 54 (2007), 1573-1583. 1, 3.4, 5.3, 3, 5.3

[12] Y. He, Q.-G. Wang, C. Lin, M. Wu, Augmented Lyapunov functional and delay-dependent stability criteria for neutral systems, Internat. J. Robust Nonlinear Control, 15 (2005), 923-933. 1, 5.5, 5, 5.5

[13] O. M. Kwon, J. H. Park, S. M. Lee, On stability criteria for uncertain delay-differential systems of neutral type with time-varying delays, Appl. Math. Comput., 197 (2008), 864-873. 5.4, 4, 5.4

[14] P.-L. Liu, A delay decomposition approach to stability analysis of neutral systems with time-varying delay, Appl. Math. Model., 37 (2013), 5013-5026. 1

[15] Y.-J. Liu, S. M. Lee, O. M. Kwon, J. H. Park, Robust delay-dependent stability criteria for time-varying delayed Lur'e systems of neutral type, Circuits Systems Signal Process., 34 (2015), 1481-1497. 1

[16] R.-Q. Lun, H.-Y. Wu, J.-J. Bai, New delay-dependent robust stability criteria for uncertain neutral systems with mixed delays, J. Franklin Inst., 351 (2014), 1386-1399. 1

[17] X.-H. Nian, H. Pang, W.-H. Gui, H.-B. Wang, New stability analysis for linear neutral system via state matrix decomposition, Appl. Math. Comput., 215 (2009), 1830-1837. 1, 5.4, 4, 5.4

[18] M.-J. Park, O.-M. Kwon, J.-H. Park, S.-M. Lee, Delay-dependent stability criteria for linear time-delay system of neutral type, Int. J. Comput. Electr. Autom. Control Inf. Eng., 4 (2010), 1602-1606. 1, 5.4, 4, 5.4

[19] F. Qiu, B.-T. Cui, Y. Ji, Delay-dividing approach for absolute stability of Lurie control system with mixed delays, Nonlinear Anal. Real World Appl., 11 (2010), 3110-3120. 1, 3.3, 3.4

[20] K. Ramakrishnan, G. Ray, Improved delay-range-dependent robust stability criteria for a class of Lur'e systems with sector-bounded nonlinearity, J. Franklin Inst., 348 (2011), 1769-1786. 1

[21] K. Ramakrishnan, G. Ray, An improved delay-dependent stability criterion for a class of Lure systems of neutral type, J. Dyn. Syst. Meas. Control, 134 (2012), 6 pages. 1, 3.4, 5.3, 3, 5.3

[22] K.-B. Shi, X.-Z. Liu, Y.-Y. Tang, H. Zhu, S.-M. Zhong, Some novel approaches on state estimation of delayed neural networks, Inform. Sci., 372 (2016), 313-331. 1

[23] K.-B. Shi, H. Zhu, S.-M. Zhong, Y. Zeng, Y.-P. Zhang, New stability analysis for neutral type neural networks with discrete and distributed delays using a multiple integral approach, J. Franklin Inst., 352 (2015), 155-176. 1

[24] R. Sivasamy, R. Rakkiyappan, Improved stability criteria for neutral type Lur'e systems with time-varying delays, Appl. Math. Lett., 38 (2014), 168-173. 1

[25] W.-Q. Wang, S. K. Nguang, S.-M. Zhong, F. Liu, New delay-dependent stability criteria for uncertain neutral system with time-varying delays and nonlinear perturbations, Circuits Systems Signal Process., 33 (2014), 2719-2740. 1

[26] W.-Q. Wang, S. K. Nguang, S.-M. Zhong, F. Liu, Novel delay-dependent stability criterion for time-varying delay systems with parameter uncertainties and nonlinear perturbations, Inform. Sci., 281 (2014), 321-333. 1

[27] M. Wu, Y. He, J.-H. She, New delay-dependent stability criteria and stabilizing method for neutral systems, IEEE Trans. Automat. Control, 49 (2004), 2266-2271. 1, 5.5, 5, 5.5

[28] Z.-G. Wu, J. H. Park, H.-Y. Su, J. Chu, Reliable passive control for singular systems with time-varying delays, J. Process Control, 23 (2013), 1217-1228. 1

[29] Z.-G. Wu, P. Shi, H.-Y. Su, J. Chu, Sampled-data synchronization of chaotic Lur'e systems with time delays, IEEE Trans. Neural Netw. Learn. Syst., 24 (2013), 410-421. 1

[30] J.-W. Xia, J. H. Park, T. H. Lee, B.-Y. Zhang, $\mathrm{H}_{\infty}$ tracking of uncertain stochastic time-delay systems: memory statefeedback controller design, Appl. Math. Comput., 249 (2014), 356-370. 1

[31] J.-W. Xia, C.-Y. Sun, B.-Y. Zhang, New robust $\mathrm{H}_{\infty}$ control for uncertain stochastic Markovian jumping systems with mixed delays based on decoupling method, J. Franklin Inst., 349 (2012), 741-769. 1

[32] L.-L. Xiong, J.-K. Tian, X.-Z. Liu, Stability analysis for neutral Markovian jump systems with partially unknown transition probabilities, J. Franklin Inst., 349 (2012), 2193-2214. 1

[33] C. Yin, S.-M. Zhong, W.-F. Chen, On delay-dependent robust stability of a class of uncertain mixed neutral and Lur'e dynamical systems with interval time-varying delays, J. Franklin Inst., 347 (2010), 1623-1642. 1

[34] C. Yin, S.-M. Zhong, W.-F. Chen, Robust $\mathrm{H}_{\infty}$ control for uncertain Lure systems with sector and slope restricted nonlinearities by PD state feedback, Nonlinear Anal. Real World Appl., 12 (2011), 501-512. 1

[35] H.-B. Zeng, J. H. Park, J.-W. Xia, S.-P. Xiao, Improved delay-dependent stability criteria for T-S fuzzy systems with time-varying delay, Appl. Math. Comput., 235 (2014), 492-501. 1, 2.1

[36] D. Zhang, L. Yu, $\mathrm{H}_{\infty}$ output tracking control for neutral systems with time-varying delay and nonlinear perturbations, Commun. Nonlinear Sci. Numer. Simul., 15 (2010), 3284-3292. 1 
[37] D. Zhang, L. Yu, Exponential stability analysis for neutral switched systems with interval time-varying mixed delays and nonlinear perturbations, Nonlinear Anal. Hybrid Syst., 6 (2012), 775-786.

[38] Z.-J. Zhao, Q.-K. Song, S.-R. He, Passivity analysis of stochastic neural networks with time-varying delays and leakage delay, Neurocomputing, 125 (2014), 22-27. 1

[39] Z.-R. Zhao, W. Wang, B. Yang, Delay and its time-derivative dependent robust stability of neutral control system, Appl. Math. Comput., 187 (2007), 1326-1332. 1

$1,5.4,4,5.4$ 